Portland State University

PDXScholar

\title{
Evaluating Mobility Impacts of Construction Work Zones on Utah Transportation System Using Machine Learning Techniques
}

Ali Hassandokht Mashhadi

University of Utah

Abbas Rashidi

University of Utah

Follow this and additional works at: https://pdxscholar.library.pdx.edu/trec_reports

Digitalrt of the Transportation Commons, Urban Studies Commons, and the Urban Studies and Planning cemmens

Newtorks know how access to this document benefits you. Logo

\section{Recommended Citation}

Mashhadi, A. H., Rashidi, A., Evaluating Mobility Impacts of Construction Work Zones on Utah Transportation System Using Machine Learning Techniques. NITC-SS-1362, Portland, OR: Transportation Research and Education Center (TREC), 2021. https://dx.doi.org/10.15760/trec.263

This Report is brought to you for free and open access. It has been accepted for inclusion in TREC Final Reports by an authorized administrator of PDXScholar. Please contact us if we can make this document more accessible: pdxscholar@pdx.edu. 


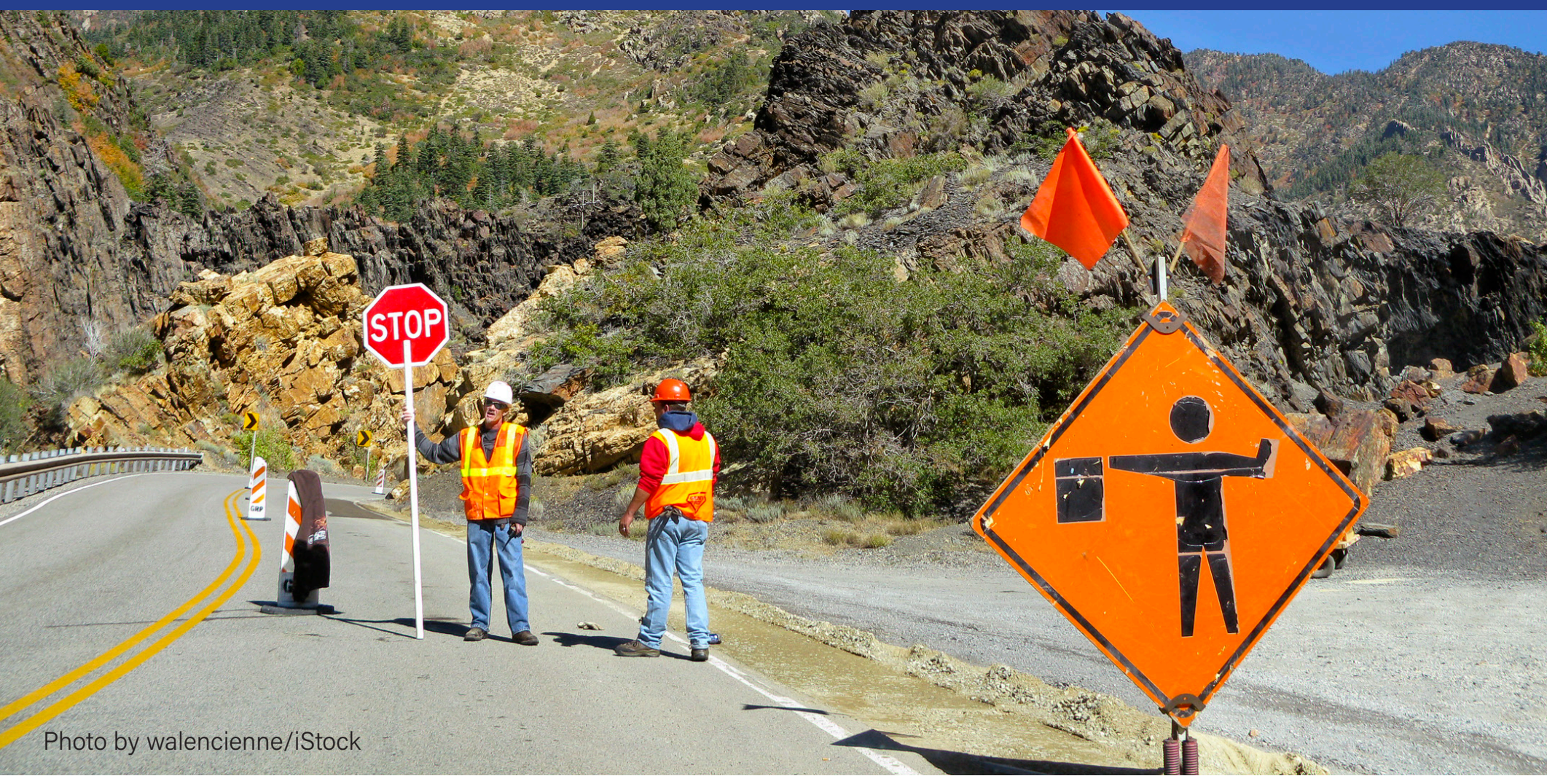

\section{Evaluating Mobility Impacts Of Construction Workzones On Utah Transportation System Using Machine Learning Techniques}

Ali Hassandokht Mashhadi Abbas Rashidi, Ph.D. 


\section{EVALUATING MOBILITY IMPACTS OF CONSTRUCTION WORK ZONES ON UTAH TRANSPORTATION SYSTEM USING MACHINE LEARNING TECHNIQUES}

Final Report

NITC-SS-1362

By

Ali Hassandokht Mashhadi, Abbas Rashidi University of Utah

for

National Institute for Transportation and Communities (NITC)

P.O. Box 751

Portland, OR 97207
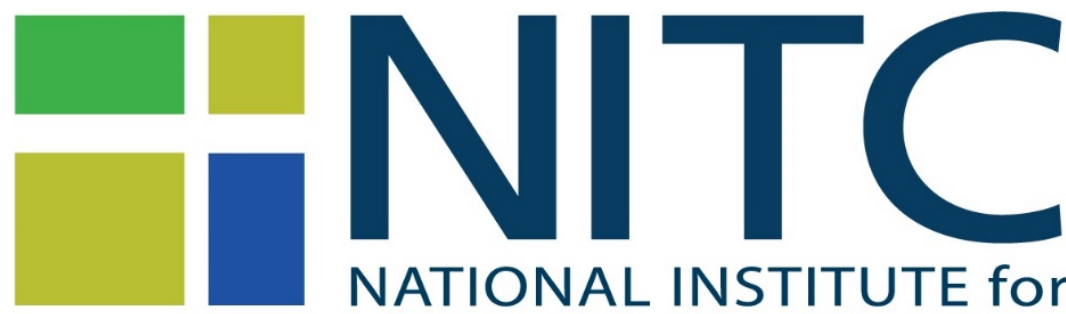

NATIONAL INSTITUTE for

TRANSPORTATION and COMMUNITIES

September 2021 


\begin{tabular}{|c|c|c|c|c|c|}
\hline \multicolumn{6}{|c|}{ Technical Report Documentation Page } \\
\hline $\begin{array}{l}\text { 1. Report No. } \\
\text { NITC-SS-1362 }\end{array}$ & \multicolumn{3}{|l|}{ 2. Government Accession No. } & \multicolumn{2}{|c|}{ 3. Recipient's Catalog No. } \\
\hline \multicolumn{4}{|c|}{$\begin{array}{l}\text { 4. Title and Subtitle } \\
\text { Evaluating Mobility Impacts of Construction Work Zones on Utah Transportation System } \\
\text { Using Machine Learning Techniques }\end{array}$} & \multicolumn{2}{|c|}{$\begin{array}{l}\text { 5. Report Date } \\
\text { September } 2021 \\
\text { 6. Performing Organization Code }\end{array}$} \\
\hline \multicolumn{4}{|c|}{$\begin{array}{l}\text { 7. Author(s) } \\
\text { Ali Hassandokht Mashhadi, https://orcid.org/0000-0002-7792-4645 } \\
\text { Abbas Rashidi, https://orcid.org/0000-0002-4342-0588 }\end{array}$} & \multicolumn{2}{|c|}{$\begin{array}{l}\text { 8. Performing Organization Report } \\
\text { No. }\end{array}$} \\
\hline \multicolumn{4}{|c|}{$\begin{array}{l}\text { 9. Performing Organization Name and Address } \\
\text { The University of Utah } \\
\text { Department of Civil and Environmental Engineering } \\
\text { 201 Presidents Circle } \\
\text { Salt Lake City, UT } 84112\end{array}$} & \multicolumn{2}{|c|}{$\begin{array}{l}\text { 11. Contract or Grant No. } \\
\text { NITC } 1362\end{array}$} \\
\hline \multicolumn{4}{|c|}{$\begin{array}{l}\text { 12. Sponsoring Agency Name and Address } \\
\text { National Institute for Transportation and Communities (NITC) } \\
\text { P.O. Box } 751 \\
\text { Portland, OR } 97207\end{array}$} & \multicolumn{2}{|c|}{$\begin{array}{l}\text { 13. Type of Report and Period } \\
\text { Covered } \\
\text { Final Report } 12 / 1 / 2019-7 / 26 / 21 \\
\text { 14. Sponsoring Agency Code }\end{array}$} \\
\hline \multicolumn{6}{|l|}{ 15. Supplementary Notes } \\
\hline \multicolumn{6}{|c|}{$\begin{array}{l}\text { 16. Abstract } \\
\text { Construction work zones are inevitable parts of daily operations at roadway systems. They have a significant impact on traffic } \\
\text { conditions and the mobility of roadway systems. The traffic impacts of work zones could significantly vary due to several } \\
\text { interacting factors such as work zone factors (work zone location and layout, length of the closure, work zone speed, intensity, } \\
\text { and daily active hours); traffic factors (percentage of heavy vehicles, highway speed limit, capacity, mobility, flow, density, } \\
\text { congestion, and occupancy); road factors (number of total lanes, number of open lanes, and pavement grade and condition); } \\
\text { temporal factors (e.g., year, season, month, weekday, daytime, and darkness); weather conditions (rainy, sunny, and snowy); } \\
\text { and spatial factors (road lane width, proximity, and number of ramps). } \\
\text { Utah Department of Transportation (UDOT) is continuously collecting and storing project-related data. Due to the significant } \\
\text { impact of work zones on traffic conditions, they are interested in evaluating the impacts of work zone attributes on mobility and } \\
\text { traffic conditions of roadway systems within the state of Utah. Such an analysis will help the UDOT personnel better understand } \\
\text { and plan for more efficient work zone operations, select the most effective traffic management systems for work zones, and } \\
\text { assess the hidden costs of construction operations at work zones. } \\
\text { To help UDOT address this problem, we propose a robust, deep neural network (DNN) model capable of evaluating the impacts } \\
\text { of the factors mentioned earlier on the mobility conditions of Utah roadway systems. DNNs can capture all the relationships } \\
\text { between input variables and output compared to traditional machine learning algorithms. The results of this project show that } \\
\text { work zone features have an important effect on the traffic condition. In the end, the performance of the model is evaluated using } \\
\text { three different measures, including R2 score, RMSE, and MAE. Comparing the measurement to previously conducted research, } \\
\text { it is the first study that has attempted to investigate the effect of work zone features on hourly traffic volume. }\end{array}$} \\
\hline \multicolumn{2}{|c|}{$\begin{array}{l}\text { 17. Key Words } \\
\text { Work zone Capacity, Traffic Prediction, Work Zone Traffic Estimation, } \\
\text { Artificial Neural Network, Non-parametric Method }\end{array}$} & \multicolumn{4}{|c|}{$\begin{array}{l}\text { 18. Distribution Statement } \\
\text { No restrictions. Copies available from NITC: } \\
\text { www.nitc-utc.net }\end{array}$} \\
\hline 19. Security Classification (of this report) & $\begin{array}{l}\text { 20. Security Classification } \\
\text { page) } \\
\text { Unclassified }\end{array}$ & f this & & of Pages & 22. Price \\
\hline
\end{tabular}




\section{ACKNOWLEDGEMENTS}

The authors acknowledge the National Institute for Transportation and Communities (NITC; grant number 1362), a U.S. DOT University Transportation Center, for funding this research. The authors also would like to thank Dr. Nikola Markovic, assistant professor at the University of Utah, for helping to guide the research.

\section{DISCLAIMER}

The contents of this report reflect the views of the authors, who are solely responsible for the facts and the accuracy of the material and information presented herein. This document is disseminated under the sponsorship of the U.S. Department of Transportation University Transportation Centers Program in the interest of information exchange. The U.S. Government assumes no liability for the contents or use thereof. The contents do not necessarily reflect the official views of the U.S. Government. This report does not constitute a standard, specification, or regulation.

\section{RECOMMENDED CITATION}

Mashhadi, A. H., Rashidi, A., Evaluating Mobility Impacts of Construction Work Zones on Utah Transportation System Using Machine Learning Techniques. NITC-SS-1362, Portland, OR: Transportation Research and Education Center (TREC), 2021. 


\section{Table of Contents}

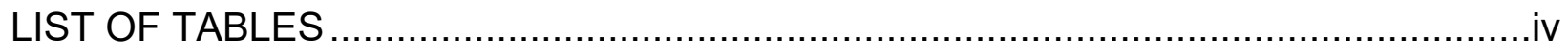

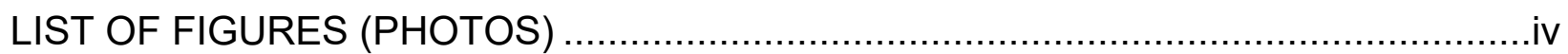

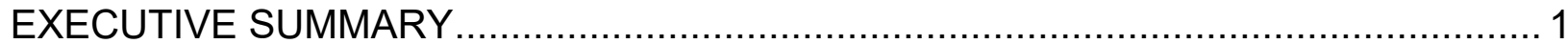

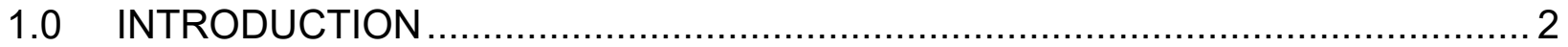

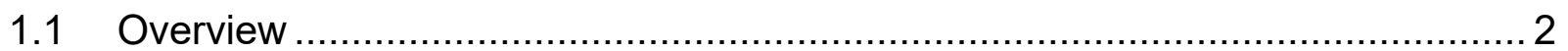

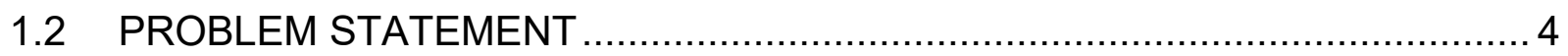

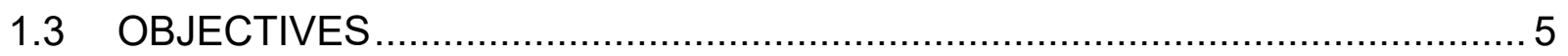

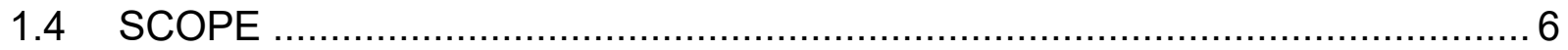

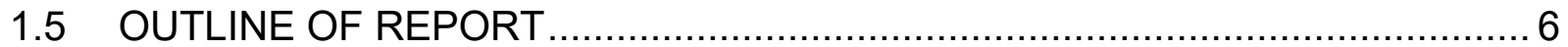

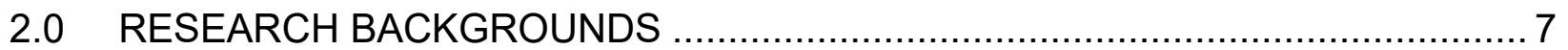

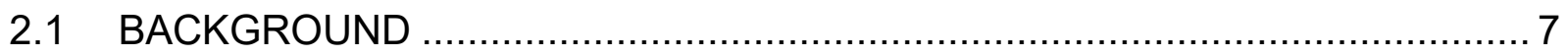

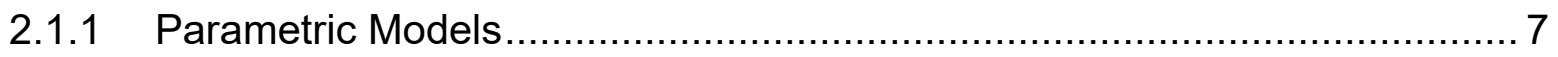

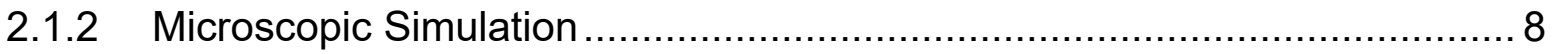

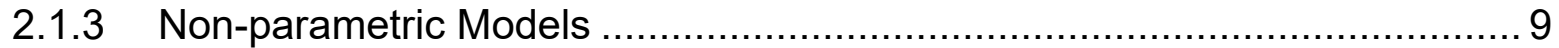

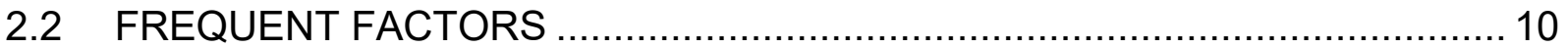

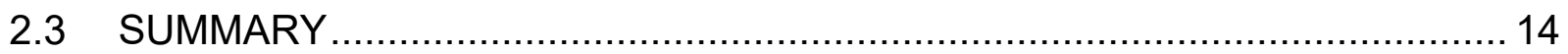

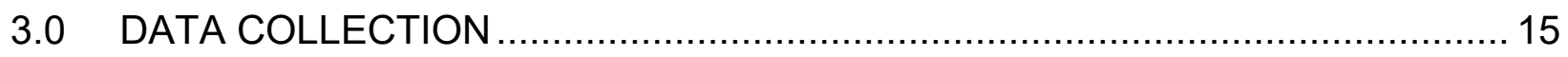

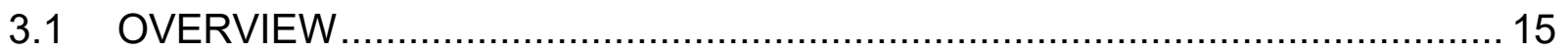

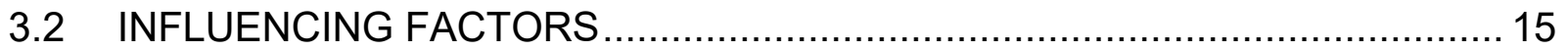

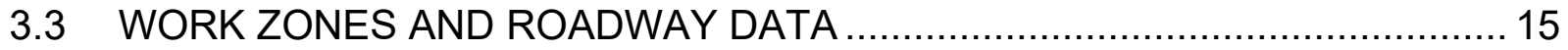

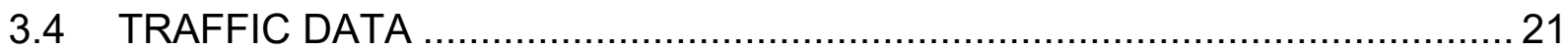

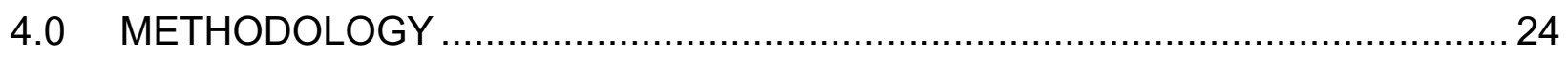

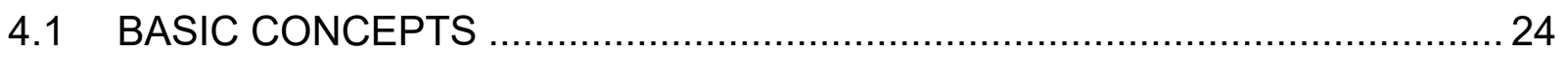

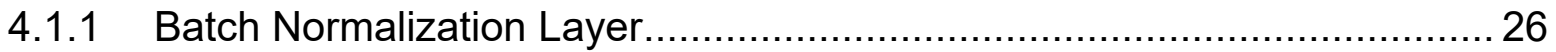

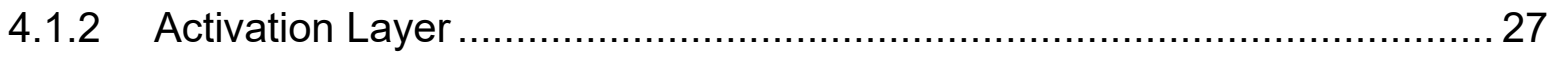

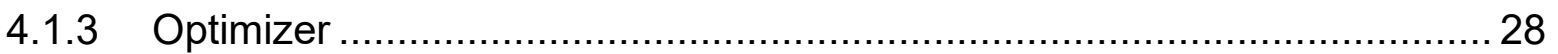

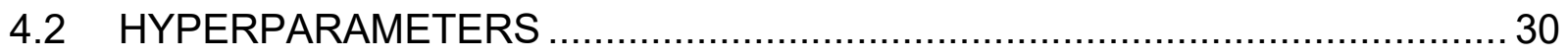

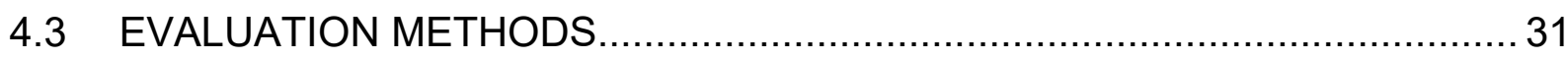

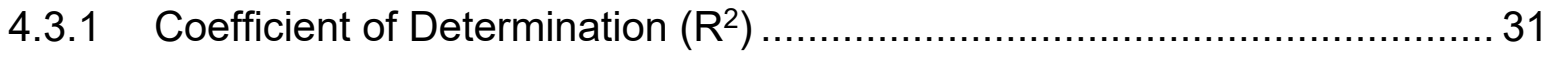

4.3.2 Mean Absolute Percentage Error (MAPE) ……...................................... 31 


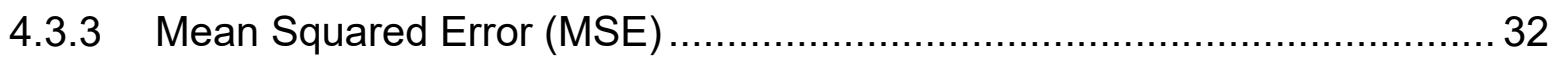

4.3.4 Root Mean Square Error (RMSE) ....................................................... 32

4.3.5 Mean Absolute Error (MAE) ......................................................... 32

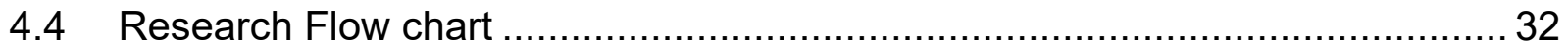

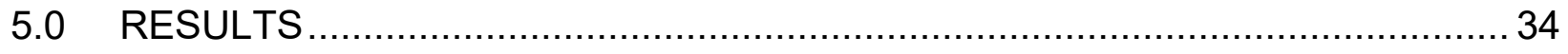

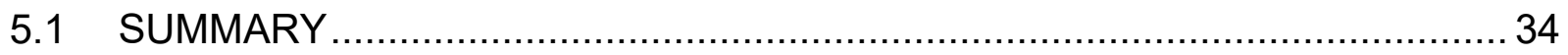

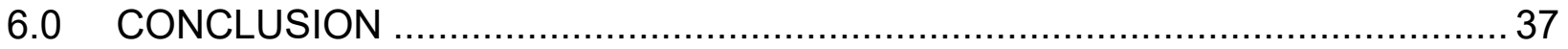

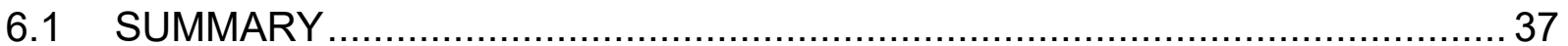

6.2 LIMITATIONS AND RECOMMENDATIONS ................................................. 37

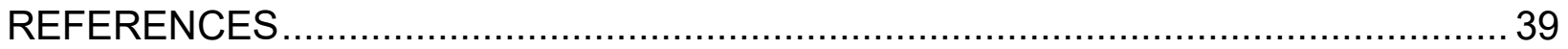




\section{LIST OF TABLES}

Table 2.1: Frequency of the Commonly Considered Factors .................................... 12

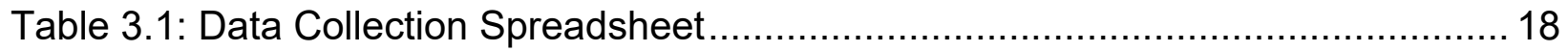

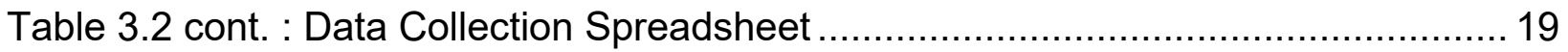

Table 3.3 cont. : Data Collection Spreadsheet ...................................................... 20

Table 3.4: Work zones Location and the Adjacent Sensors ...................................... 22

Table 3.5: Extracted Traffic Data using the PeMS Database ..................................... 23

Table 4.1: Considered Training Parameters Based on Hyperparameter Tuning ............ 30

\section{LIST OF FIGURES (PHOTOS)}

Figure 1.1: U.S. Congestion Problem Trends in Recent Years ................................. 2

Figure 1.2: PeMS Dataset for Extracting Traffic Data.............................................. 4

Figure 1.3: Location and Detailed Information on Projects in Utah .............................. 5

Figure 2.1: VISSIM Model Calibration Procedure [19] .............................................. 9

Figure 2.2: Framework For Work Zone Capacity Prediction [26] ................................ 10

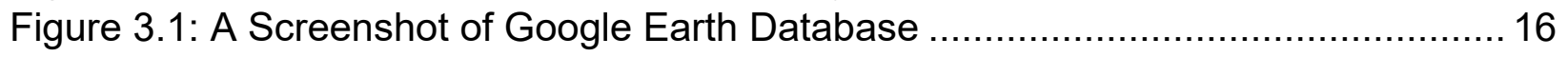

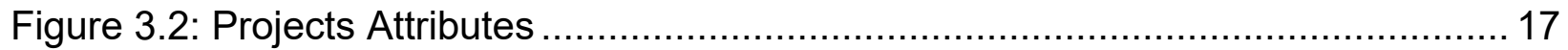

Figure 3.3: Location of Sensors on the Roadways of the State ................................... 21

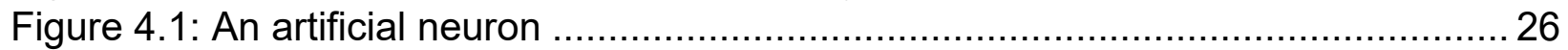

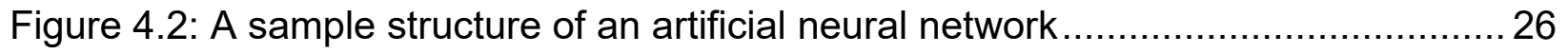

Figure 4.3: Structure of the Fully Connected Neural Network ..................................... 30

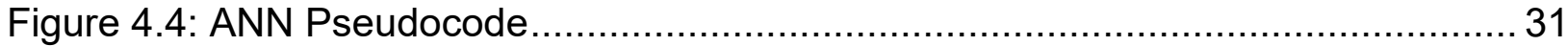

Figure 4.5: Work Zone Traffic Estimation Methodology............................................ 33

Figure 5.1: Loss Function and R2 score diagram for 20 Epochs and Batch Size of 200

Figure 5.2: MAE and RMSE metrics for 20 Epochs and Batch Size of 200................. 34

Figure 5.3: Loss Function and R2 score diagram for 20 Epochs and Batch Size of 500

Figure 5.4: MAE and RMSE metrics for 20 Epochs and Batch Size of $500 \ldots \ldots \ldots \ldots \ldots \ldots . . . . .35$

Figure 5.5: Graphical comparison of the model performance and the actual data ........ 36 


\section{EXECUTIVE SUMMARY}

Construction work zones play a significant role in traffic delays and congestion on state roadways. The roadway capacity is decreased due to fewer traffic lanes, narrower lanes, and work zone speed limits. To this end, accurate prediction of work zones' capacity is of utmost importance to transportation agencies. Thanks to the advancement of technologies, there are many traffic sensors installed on roadways. The considerable amount of traffic data collected by these sensors at every time of the day provided researchers with the chance to investigate possible influential factors on road traffic. The traffic impacts of work zones could significantly vary due to several interacting factors such as work zone factors (work zone location and layout, length of the closure, work zone speed, intensity, and daily active hours); traffic factors (percentage of heavy vehicles, highway speed limit, capacity, mobility, flow, density, congestion, and occupancy); road factors (number of total lanes, number of open lanes, and pavement grade and condition); temporal factors (e.g., year, season, month, weekday, daytime, and darkness); weather conditions (rainy, sunny, and snowy); and spatial factors (road lane width, proximity, and number of ramps). Utah, as one of the pioneer states in using novel techniques in traffic management, is interested in evaluating the impacts of those factors on mobility and traffic conditions of roadway systems within the state.

To address work zones' capacity on roadways, this project proposed an artificial neural network model based on the collected data by Utah transportation agencies. In order to determine the most influential factors of work zones, a comprehensive literature review has been conducted on 70 previously published papers. Lane width, work zone length, project duration, time of day, day of the week, and heavy vehicle percentage are among the most common factors considered in the literature. The suggested neural network model is trained and evaluated on around 400,000 data points collected from about 80 projects on Utah roadways. Based on the collected data from various resources, a fourlayer neural network has been developed with 256 neurons in each hidden layer. The developed model is trained on $70 \%$ of the data and is evaluated using the other $30 \%$, divided into $15 \%$ of validation and $15 \%$ of test set. Numerical results of a random seed show consistent outperformance of the proposed model, with an R2, RMSE, and MAE being $0.98,158$, and 101, respectively. Based on the results of this project, future studies could be carried out using the probe vehicle data to improve the model's performance by decreasing the RMSE, MAPE, and MAE values. 


\subsection{INTRODUCTION}

\subsection{Overview}

The increased load on road infrastructures due to population growth has resulted in an increment in the need for road maintenance and reconstruction activities, including increasing the line number, replacing the pavement, and adding or removing traffic signs. Road maintenance activities usually require lane closures that will cause increased traffic crashes and delays because of reduced capacity. According to the urban mobility report [1], traffic congestion in 2017 caused 8.8 billion hours of travel delay, with 3.3 billion gallons more fuel consumption, equal to a congestion cost of $\$ 179$ billion. Figure 1.1 shows the trend of traffic congestion effects on public loss.

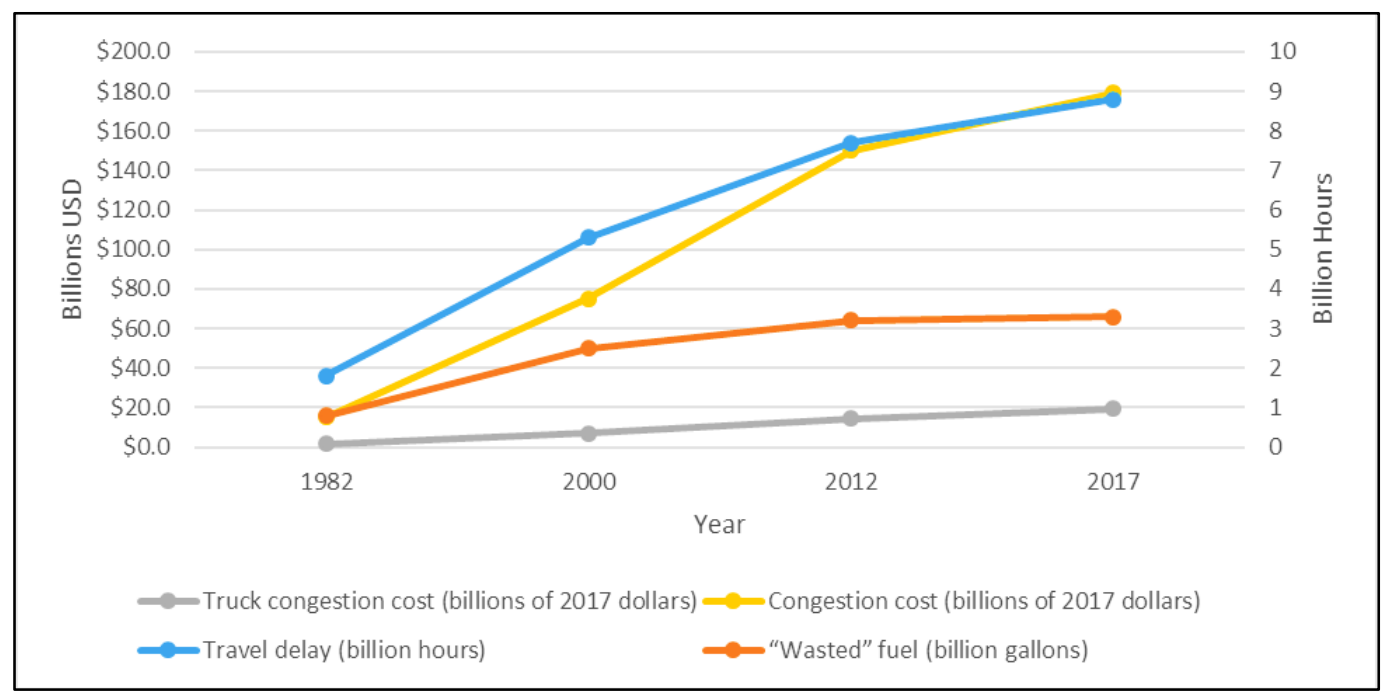

Figure 1.1: U.S. Congestion Problem Trends in Recent Years

Construction work zones are among the main reasons for traffic congestion and nonrecurring delays in every transportation network. Other reasons for nonrecurring delays are crashes, weather conditions, and disabled vehicles. However, the top three causes of nonrecurring delays are incidents ( $25 \%$ of congestion), work zones ( $10 \%$ of congestions), and adverse weather conditions (15\% of congestion) [2]. Appropriate management of temporary disruptions could mitigate their effect.

The effect of work zones as one of the causes of traffic delays could further diminish by deploying a precise traffic prediction model. Accurate work zone traffic prediction could equip drivers to handle any upcoming delays or congestion in their travel. Travelers are especially more sensitive to unpredicted congestion because of tightly scheduled daily activities. Additionally, work zone traffic prediction providing agencies with adequate information helps them in resource allocation according to the congestion risk of each specific road [3]. Moreover, because of the dynamic nature of the traffic network, precise work zone impact prediction could alleviate traffic congestion, directly impacting fuel consumption and air pollution [4]. 
Many studies have investigated the effect of work zones on traffic conditions and road capacity. Such studies accounted for a wide range of parameters that may affect capacity reduction. Depending on the work zone's nature and configuration, they could be classified into various groups, such as work zone configuration, roadway conditions, work activity characteristics, and environmental conditions. Work zone features, such as work zone length, work time, work zone speed limit, heavy vehicle percentage, work zone grade, work intensity, road type, number of opened/closed lanes, and lane width, are among the work zone features that could affect roadway capacity.

The simplicity of implementing traditional approaches is why many works of literature have employed them in their studies. Traditional approaches use the deterministic queuing theory based on the relationship between approaching traffic volume and limited capacity. However, several interacting variables might contribute to traffic conditions around work zones, which cannot be represented in a simple mathematical function. To this end, the predicted capacities are usually inaccurate because of the simplified conditions.

To improve the effectiveness of work zone traffic management plans (TMP), novel and automated approaches are required to identify the relationship between work zone parameters and traffic characteristics such as delay time, capacity, and queue length. State departments of transportation (DOTs), especially the Utah Department of Transportation (UDOT), collect various data types related to work zone operations and are interested in evaluating the potential impacts of these variables on traffic and mobility conditions at the roadway system. One potential method of finding traffic patterns and evaluating the effect of work zones on traffic parameters is the use of machine learning techniques. Machine learning algorithms, such as decision trees, ensemble learning, support vector machine (SVM), and artificial neural network (ANN), have been widely used for prediction work zones capacity.

Another possible method of investigating the effect of work zones on roadway traffic Is using microscopic simulation models. One of the advantages of using a simulation model is determining the impact of each work zone feature on the road capacity [5], [6]. However, the need for specialized software packages, model calibration, and computational resources are some of the downsides of these models. A few examples of these softwares are CORSIM and VISSIM. With the availability of more data each day, the better accuracy and the less need for technologies are some of the reasons pushing researchers to use data-driven approaches, such as machine learning algorithms.

This project aims to develop a neural network-based model to estimate the work zone traffic volume on Utah's freeways. It is worth noting that the developed model can estimate work zone traffic in areas without any traffic sensors. 


\subsection{PROBLEM STATEMENT}

Recently, DOTs in the United States have started making landmark decisions on implementing data-driven approaches to daily state problems. However, due to regionbased developed models, transportation divisions cannot select the right technology unless they know whether that technology can meet their requirements. Due to the higher accuracy of machine learning methods, some of the pioneer DOTs, such as UDOT, in the United States have begun using them in their decision-making.

Fortunately, UDOT continuously collects and records all necessary data required for this project. UDOT also has access to two data sources, the freeway Performance Measurement System (PeMS) and iPeMS. The iPeMS website provides statewide HERE probe data, and freeway PeMS provides data from the UDOT traffic management system. Freeway PeMS data is collected from radar, loops, and microloops. This data can later be preprocessed and prepared to be utilized in the model. Figure 1.2 shows the interface of PeMS and the recorded traffic data on freeway I15 at a specific time.

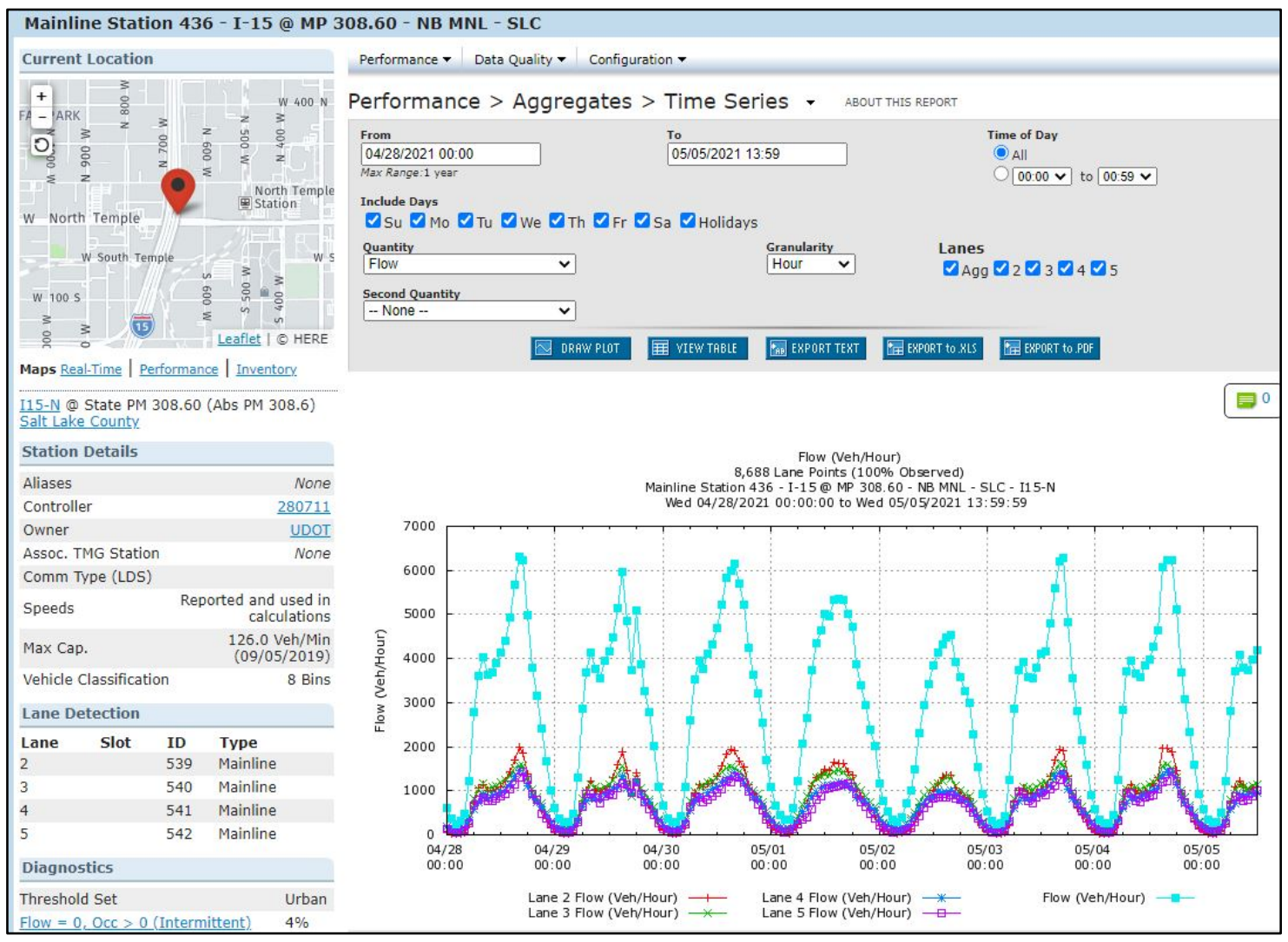

Figure 1.2: PeMS Dataset for Extracting Traffic Data 
Moreover, UDOT stores work zone data in their databases, and those databases are updated frequently. Figure 1.3 shows the website that shows the completed and ongoing projects in Utah.

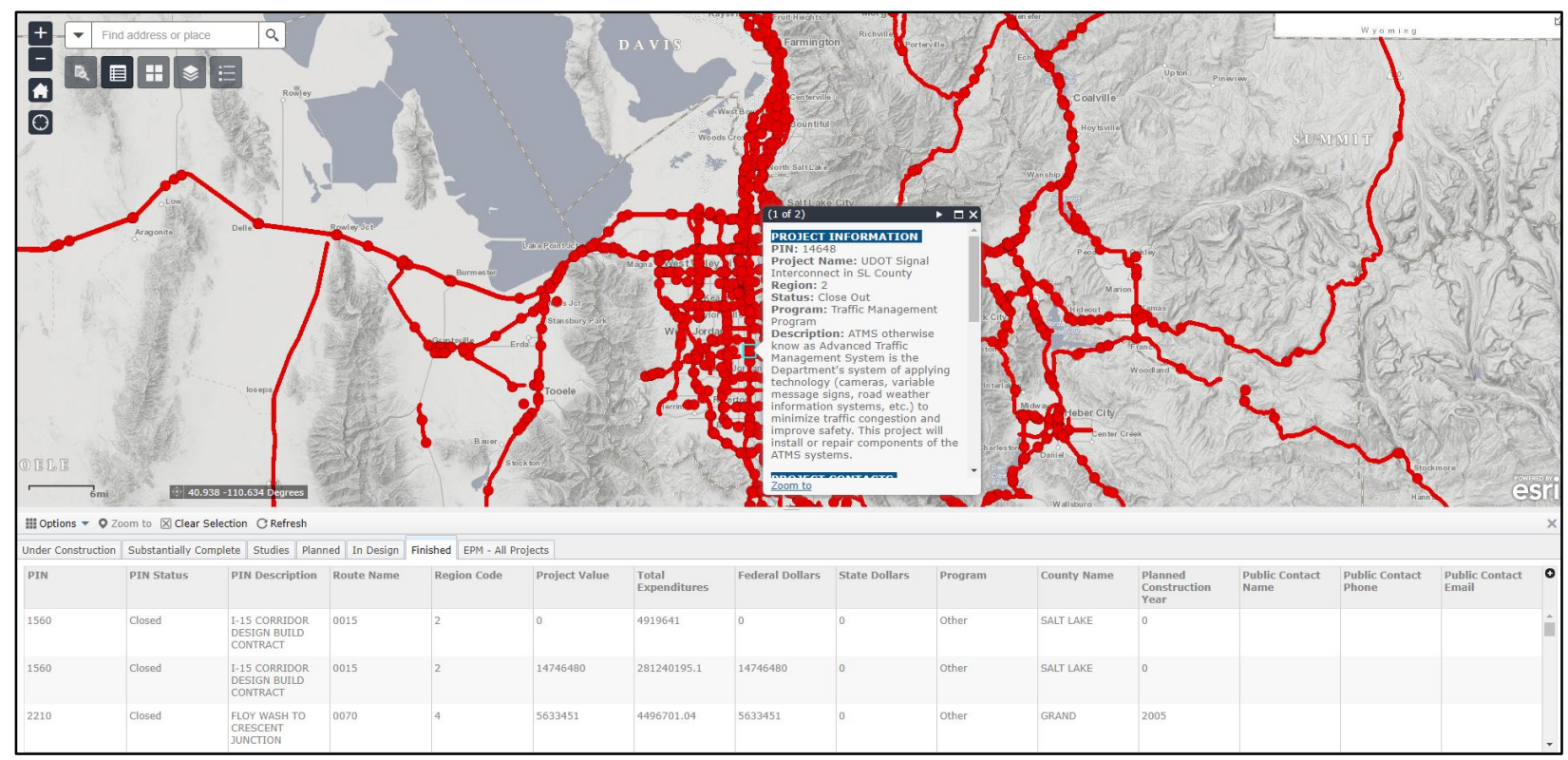

Figure 1.3: Location and Detailed Information on Projects in Utah

A deep learning model could be developed using the traffic data collected from PeMS and the work zone features from the UDOT project database. The output data could further assist the decision-makers in assessing the most effective traffic management systems at work zones, evaluating the hidden costs of construction operations at the work zone, and better scheduling for work zone-related projects. This system is based on a deep neural network and can consider different factors affecting the traffic conditions near the work zones.

\subsection{OBJECTIVES}

Many approaches are available for predicting traffic and road capacity within work zones. While the output is similar for all of them, the considered parameters and levels of accuracy may differ. Also, one approach may work better than the other one based on the existing conditions. Despite the growing popularity of data-driven approaches, such as machine learning algorithms, and their ease of use and low-cost technology, it has not been considered as a practical tool within UDOT divisions. The primary purpose of this project is to address traffic estimation within work zones and explore the feasibility of using data-driven approaches as an alternative technology to the existing traditional methods. 


\subsection{SCOPE}

This research evaluates the mobility effect of work zones within the state of Utah. The scope of this project covers the following subjects:

- Most important features of work zones

- Traffic impact of work zones

- Traffic estimation of roadways within the work zone area

The project was conducted within these areas because the existing literature had considered different work zone features in their developed models. Therefore, finding the most effective and common features is of utmost importance for developing any model. Moreover, all of the conducted projects in this research area are related to a specific city or state. Since driving rules and drivers' behaviors within each zone are different from another zone, it is impossible to use the existing models for the state of Utah. Furthermore, there is no study investigating the effect of work zones on hourly traffic. Most of the studies in this field focused on work zone capacity prediction. To this end, this project developed a machine learning model based on the work zone features and traffic data collected from Utah's freeways.

\subsection{OUTLINE OF REPORT}

- Introduction

- Research Background

- Data Collection

- Methodology

- Results

- Conclusion 


\subsection{RESEARCH BACKGROUNDS \\ 2.1 BACKGROUND}

Construction work zones play an important role in delays occurring on roadway systems. Therefore, many studies have been conducted to determine the effect of work zones on roadway capacity. Moreover, to facilitate the process, the Highway Capacity Manual is provided to calculate the work zone capacity. Generally, the existing literature in the field of work zone traffic prediction could be categorized into three main groups: parametric, non-parametric, and microscopic simulation.

\subsubsection{Parametric Models}

The early studies in this field attempted to suggest mathematical models for work zone capacity. The developed models are based on the work zone attributes, road features, and collected data from various implemented projects. Based on the data collected from 33 work zones in Texas between 1987 and 1991, Krammas and Lopez [7], as one of the pioneers in this field, suggested a deterministic model for short-term work zone capacity estimation. Specifically, the model,

$$
C=(1600+I-R) \times f_{H V}
$$

estimates capacity $C$ by accounting for adjustment factors for work intensity $I$, presence of the ramp $R$, and heavy vehicle percentage $f_{H V}$. Racha et al. [8] developed a parametric model by considering speed-flow relationships using collected data from 22 work zone sites in South Carolina. The results of their study show that 1,550 passenger cars per hour per lane (pcphpl) is a reasonable estimation for a two-to-one lane closure configuration.

Long-term and short-term work zones have different capacities. Therefore, it is not ideal to use short-term models for long-term work zones capacity estimation. Various models have been proposed in the literature based on the collected data on long-term work zones. Al-Kaisy and Hall [9] developed a multiplicative model for capacity estimation in long-term work zones. The collected data are from six sites in Ontario, Canada. The results of this study depicted that heavy vehicle percentage has the most effect on the road capacity. The model,

$$
C=C_{b} \times f_{H V} \times f_{d} \times f_{w} \times f_{s} \times f_{r} \times f_{l} \times f_{i}
$$

estimates capacity $C$ by accounting for adjustment value for based work zone capacity $C_{b}$, heavy vehicle percentage $f_{H V}$, driver population $f_{d}$, work activity $f_{w}$, location of lane closure $f_{s}$, presence of rain $f_{r}$, light conditions $f_{l}$, non-additive interactive effects $f_{i}$.

In order to capture the uncertainties associated with the traffic parameters and present a more reliable capacity estimation, many studies have integrated probability distributions of parameters into their developed model. Using the probabilistic speed- 
flow relationship, Weng and Yan [10] proposed a work zone capacity estimation model based on the recorded data in Singapore. Based on this study, an increase in the geometrical alignment will result in decreasing the capacity. However, the work zone capacity increases with an increase in speed limit. In the next section, a literature review of non-parametric studies will be presented. Weng and Yan [11] also proposed another probability model for work zones in the United States. Their study provides traffic engineers with a range of work zone capacity at a corresponding prediction band at a specific confidence level. However, one of the downsides to this study is that the model was developed based on the US data; therefore, it may not be applicable to other countries due to differences in driving behavior and rules. Weng et al. [12] suggested a stochastic model to estimate the uncertainty of work zone capacity. The proposed model could be applied to evaluate travel time reliability. Some of the limitations related to this study are: 1) The limited examination of explanatory factors other than those that were considered, 2) The assumption of a linear relationship between capacity and other variables.

\subsubsection{Microscopic Simulation}

Another well-known approach for estimating the work zone capacity is the microscopic simulation. Using this approach, researchers could determine the effect of each work zone or road attribute on the capacity. Specialized software, model calibration, and expensive computations are some of the disadvantages of using simulation models. The most popular software packages of microscopic simulation are CORSIM, VISSIM, and PARAMICS. Ping and Zhu [13] used CORSIM to estimate the work zone capacity under different conditions. In another study, Chatterjee et al. [14], using VISSIM, proposed a work zone capacity model by considering drivers' behaviors. Also, Wen built a PARMICS simulation model for a prototypical freeway work zone in a connected vehicle environment. Four different models were constructed for the travel time analysis, including linear regression, multivariate adaptive regression splines (MARS), stepwise regression, and elastic net. The results show that the different modeling approaches have similar performance in terms of the Root of Mean Square Error (RMSE) [15]. In another study, Das and Chattaraj developed a work zone traffic simulation model using cellular automata. The results of their study showed that the lane drop not only produces a jam in the blocked lane as well as in the bypass lane [16]. Model calibration is an inevitable part of simulation models. Therefore, many researchers have suggested various calibration methods. Yeom et al. [17] proposed a methodology to calibrate work zone capacity models. In order to pave the way for future studies, Kan et al. [18] suggested a procedure for calibrating VISSIM models. Figure 2.1 shows the suggested procedure.

Since parametric and simulation models only consider a limited number of work zone features and road attributes, they usually have poor performance in estimation work zone capacity. Moreover, they only present a crisp number as the work zone capacity, but the capacity could vary based on the time of day, day of the week, and other contributing features. To this end, non-parametric models were developed to overcome 
the disadvantages of the parametric and simulation models. Since non-parametric models are based on data collected from actual work zone sites, their estimations are more realistic and have better accuracy.

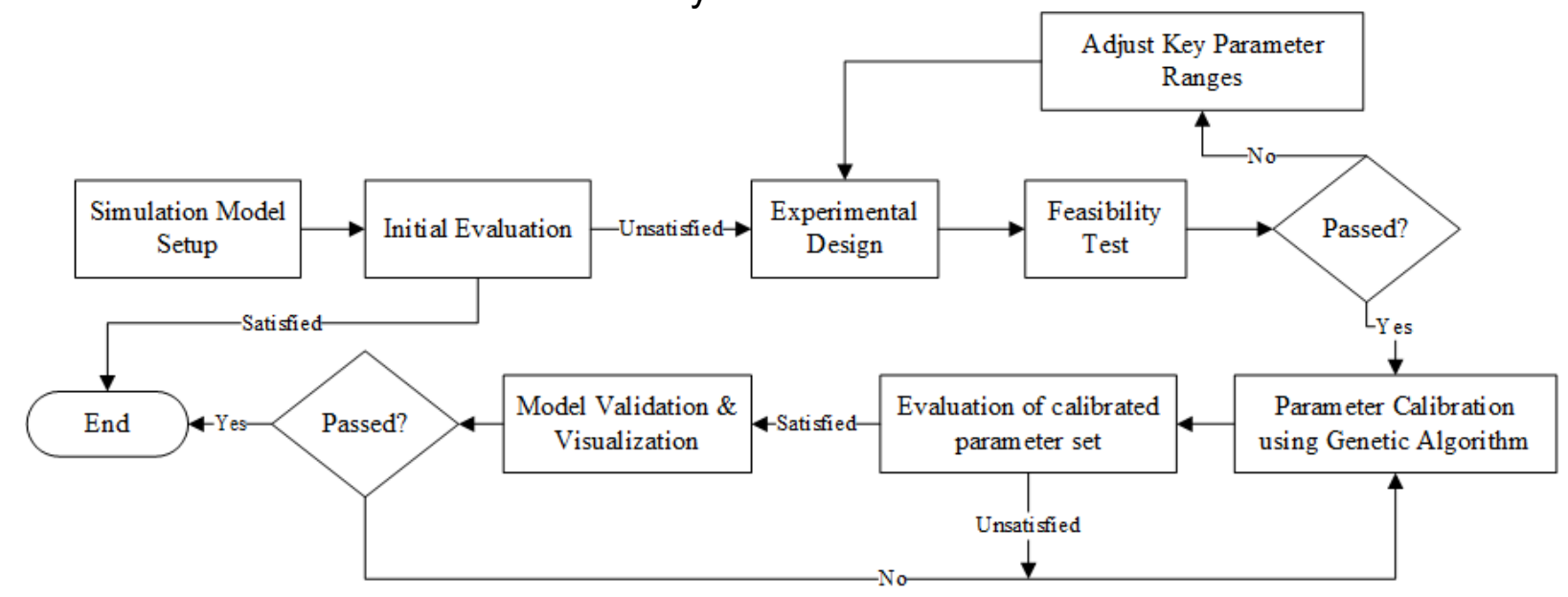

Figure 2.1: VISSIM Model Calibration Procedure [19]

\subsubsection{Non-parametric Models}

Different machine learning approaches have been adopted by researchers and applied to various transportation fields. Work zone capacity estimation is not an exception. Decision trees, ensemble learning, support vector machine (SVM), and neural network are among the most common methods. Based on the collected data from 14 cities and states, Weng and Meng [20] developed a decision tree to estimate the freeway work zone capacity. Better accuracy with no need for adjustment factors are the advantages of using this method. However, decision trees are vulnerable to small changes or any noises in the training set. Using the same dataset, Weng and Meng [21] developed an ensemble learning model using 105 individual trees to cover the disadvantages of decision trees.

Some studies used more novel techniques, such as SVM and artificial neural networks. Adeli and Jiang [22] developed a neuro-fuzzy model by considering 17 different parameters. The dataset was based on the collected data from seven cities and states in the United States. In another study, Karim and Adeli [23], considering 11 work zone factors, developed a neural network for work zone capacity estimation. A radial basis function was employed to develop deterministic results.

In order to compare the results of different models, Hou et al. [24] developed four different models: random forest, baseline predictor, regression tree, and neural network. The results of their study showed that random forest outperformed the other three models. Moreover, one of the most important findings of their study is that the work zone features do not affect the traffic flow characteristics.

To improve the results of previous researches, some studies combined different algorithms. However, all the previously developed models result in a single value as 
their outputs. In other words, they do not consider probability distributions and confidence intervals in their models. Bian and Ozbay [25] developed a Bayesian neural network with black-box variational inferences (BBVI) technique and a regular artificial neural network with Monte Carlo (MC)-dropout technique. The proposed models are capable of capturing the uncertainties associated with capacity estimation models. Figure 2.2 shows the framework for work zone capacity prediction based on nonparametric and parametric models. The framework consists of three main phases. i) Data Collection Phase: data can be collected using different technologies such as sensors, radars, cameras, vehicle-mounted GPS, and WiFi. ii) Prediction Phase: the collected data in the first phase could further be analyzed to extract more information on drivers' behaviors. iii) Application Phase: the results of the previous phase could be used in traffic management, transportation planning, and navigation.

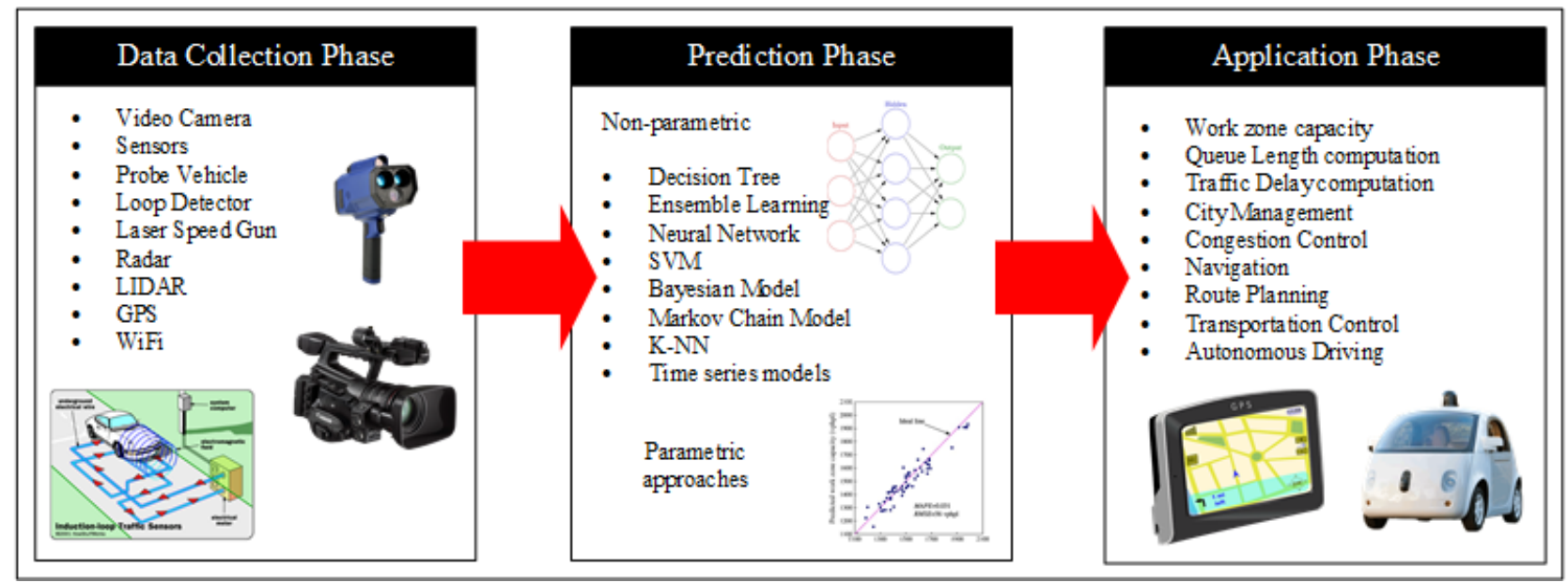

Figure 2.2: Framework for Work Zone Capacity Prediction [26]

\subsection{FREQUENT FACTORS}

Numerous studies have been performed to model freeway work zone capacity using site conditions. These studies accounted for a wide range of factors that may influence capacity reduction. Depending on the work zone's nature and configuration, these factors can be categorized into multiple groups: work zone configuration, roadway conditions, work activity characteristics, and environmental conditions [27]. Among almost 40 factors that were considered in the literature, the following were identified as the most frequent ones:

1. Heavy vehicle percentage

3. Work zone grade

5. Work zone intensity

7. Road type (Rural/ Urban)

9. Number of opened lanes
2. Lane width

4. Lane closure location (Left/ Right)

6. Work zone length

8. Weather condition (Clear/ Rain)

10. Driver composition and population 
11. Number of closed lanes

13. Work zone duration (Short/Long)

15. Work time (Day/ Night)

17. Weekday
12. Ramp (Yes/ No)

14. Work zone speed limit

16. Normal speed

18. Lateral clearance

Table 2.1 summarizes the most frequently considered factors and the outputs of the models. Some studies only focused on capacity estimation, while a couple of them considered multiple outputs simultaneously. Results show that heavy vehicle percentage, work zone grade, work zone intensity, and the number of opened and closed lanes are among the most frequent factors considered in previous studies. It is expected that a higher percentage of heavy vehicles will reduce the short- and longterm work zone capacity due to occupying more space and moving slower. Also, as the work intensity is increased from light to heavy, the work zone capacity will decrease. The most common influencing factors in the simulation-based studies include lane configuration, volume distributions, the distance of signs upstream of the work zone, presence of trucks, and rubbernecking. 
Table 2.1: Frequency of the Commonly Considered Factors

\begin{tabular}{|c|c|c|c|c|c|c|c|c|c|c|c|c|c|c|c|c|c|c|}
\hline \multirow[b]{2}{*}{ Author } & \multicolumn{18}{|c|}{ Input Variables } \\
\hline & 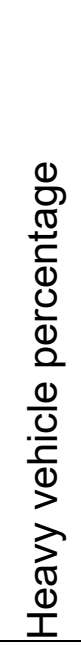 & 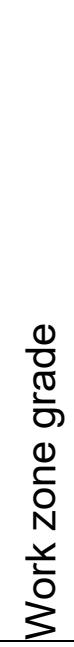 & 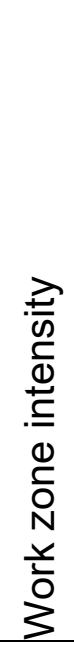 & 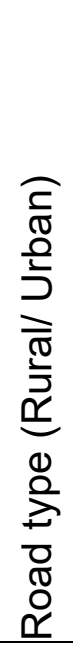 & 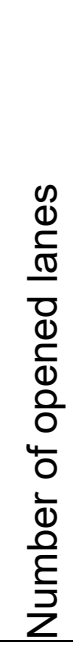 & 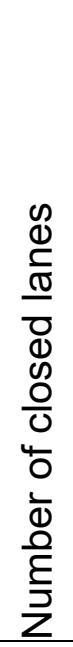 & 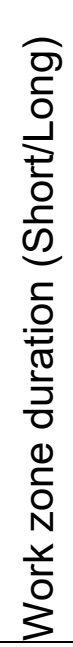 & 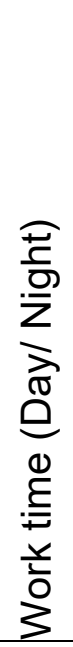 & 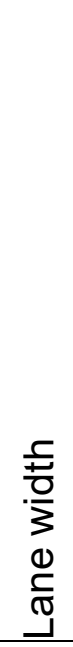 & 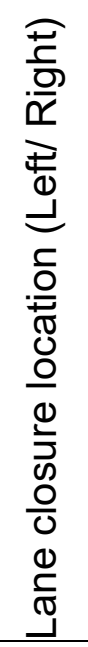 & 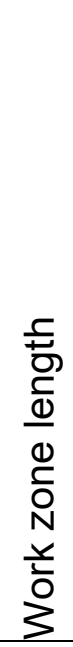 & 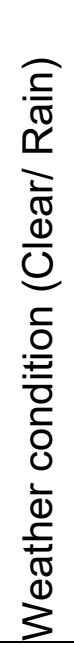 & 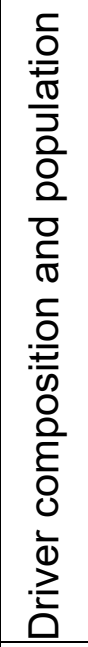 & 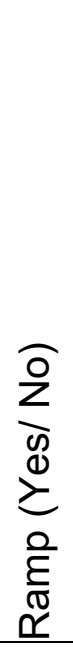 & 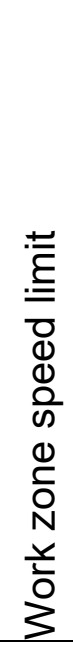 & 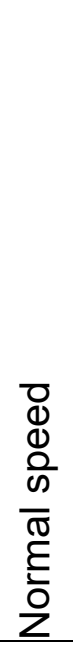 & $\begin{array}{l}\vec{d} \\
\frac{0}{0} \\
\frac{y}{0} \\
\stackrel{0}{3}\end{array}$ & 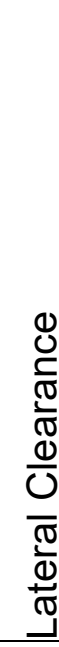 \\
\hline Abrams and Wang [28] & * & & & & & & $*$ & & $*$ & & & & & & & & & $*$ \\
\hline Dudek and Richards [29] & & & & & * & * & & & & & & & & & & & & \\
\hline Krammes and Lopez [7] & * & & * & & * & & & & & & & & & * & & & & \\
\hline Dixon [30] & & & * & & * & * & & * & & & & & & & & & & \\
\hline Dixon et al. [31] & & & * & & * & * & & * & & & & & & & & & & \\
\hline Yi [32] & * & & * & & * & * & & & & * & & & & & * & & & \\
\hline Al-Kaisy et al. [33] & * & * & & & & & & & & & & * & & & & & * & \\
\hline Maze et al. [34] & & & & & * & * & & & & & & & & & & & & \\
\hline Kim et al. [35] & * & * & * & & & * & & & & * & * & & & & & & & * \\
\hline Venugopal and Tarko [36] & * & & & & & & & & & * & & * & & & & & & \\
\hline Karim and Adeli [23] & * & * & * & & * & * & & * & * & & * & & & * & * & & & \\
\hline Adeli and Jiang [22] & * & * & * & * & * & * & * & * & * & * & * & * & * & * & * & & * & \\
\hline Al-Kaisy and Hall [9] & * & & * & & & & & * & & * & & * & * & & & & * & \\
\hline Benekohal et al. [37] & * & & * & & & & & & * & & & & & & * & & & * \\
\hline Benekohal et al. [38] & * & & * & & & & & & * & & & & & & $*$ & & & * \\
\hline Sarasua et al. [39] & * & & * & & * & & & & & & & & & & & & & \\
\hline
\end{tabular}




\begin{tabular}{|c|c|c|c|c|c|c|c|c|c|c|c|c|c|c|c|c|c|c|}
\hline Arguea [40] & * & & & & & & & & & & * & & & & & * & & \\
\hline Sarasua et al. [41] & * & & * & * & * & & & & & & * & & & & & & & \\
\hline Ping and Zhu [13] & * & * & & & * & * & & & & & & & & & & * & & \\
\hline Racha et al. [8] & * & & * & & * & & & & & * & * & & & & & & & \\
\hline Hicks et al. [42] & & & & & * & * & & * & & & & & * & & & & & \\
\hline Hicks et al. [43] & & & & & * & * & & * & & & & & * & & & & & \\
\hline Batson et al. [44] & * & & * & * & * & * & & & & & & & & * & & * & & \\
\hline Notbohm et al. [45] & * & & & & * & * & & & * & * & * & & * & & * & & & \\
\hline Weng and Meng [20] & * & * & * & * & * & * & * & * & * & * & * & * & * & * & * & & & \\
\hline Heaslip et al. [46] & & & & & * & * & & & & & * & & & & & * & & * \\
\hline Bham and Khazraee [47] & & & & & * & * & & & * & * & * & & & & & & & \\
\hline Weng and Meng [21] & * & * & * & * & * & * & * & * & * & * & * & * & * & * & * & & & \\
\hline Ortiz [48] & & & * & & * & & * & & * & * & * & * & & & & & & \\
\hline Weng and Yan [10] & & * & & & & & & & & & & & & & * & & & \\
\hline Hou et al. [24] & & & * & & * & * & * & * & & * & * & & & & * & & * & \\
\hline Weng and Meng [49] & * & * & & & & & & & & & * & & & & * & & & \\
\hline Weng and Yan [11] & * & & * & * & * & * & * & * & & * & & & & & & & & \\
\hline Heiden and Geistefeldt [50] & * & * & & * & * & * & & * & * & * & & & & & & & * & \\
\hline Stromgren and Olstam [51] & * & & & & & * & & & * & & * & & * & & & & & \\
\hline Weng et al. [12] & * & & * & * & * & * & * & * & & * & & & & & & & & \\
\hline Bae et al. [52] & & & & & * & * & & * & & & * & * & & & & & * & \\
\hline Lu et al. [53] & & & * & & * & * & & * & & & & & & * & * & * & & \\
\hline Bian and Ozbay [54] & * & & * & & * & * & * & * & & & & & & & & & & \\
\hline
\end{tabular}




\subsection{SUMMARY}

Based on the literature, different approaches have been proposed for estimating work zone capacities. Although parametric approaches are widely used by both federal and state transportation agencies, due to their simplicity and ease of implementation, it is challenging to model work zone capacity "in the closed-form" as attempted with models (2-1) and (2-2). Specifically, these models do not account for interactions between influencing factors [12]. On the other hand, non-parametric approaches can extract nonlinearity or higher-order interactions of work zone features and work zone capacity. The need for a large amount of data for training, validation, and testing is one of the drawbacks of non-parametric models. Moreover, they may fall short when applied to scenarios that were not observed historically.

It is often difficult to measure the effect of some features, such as driving behavior, the distance of warning signs, and merge strategies in the parametric and non-parametric models. Therefore, simulation-based methods have been introduced to determine the impact of these features on traffic flow and better understand future situations. Requiring computational resources, specialized software, and model calibration are some of the drawbacks of simulation-based models.

The method selection depends on various criteria. However, one of the most important factors is the amount of available data. Both parametric and non-parametric methods are data-driven approaches; however, the choice between the two may depend on the available number of data points. Since non-parametric models require a validation dataset for hyperparameter tuning as well as test data, they typically require more data points than parametric models. However, if the amount of data is large enough, nonparametric methods are expected to provide higher estimation accuracy than parametric models. 


\subsection{DATA COLLECTION \\ 3.1 OVERVIEW}

The idea behind the suggested approach is to train a neural network model to learn the relation between traffic volumes and various influencing factors of work zones. This process would enable agencies to use the model and estimate volumes at locations with no traffic sensors. Accordingly, the proposed work regresses hourly traffic volumes from traffic sensors along Utah's freeways to explanatory variables obtained from various data sources (e.g., road characteristics, work zone features). The remainder of this section provides a brief description of the most influential factors of work zones and the data needed to train the model.

\subsection{INFLUENCING FACTORS}

Based on the literature review, the following 18 factors are among the most common factors considered in the previously conducted research.

1. Heavy vehicle percentage

3. Work zone grade

5. Work zone intensity

7. Road type (Rural/ Urban)

9. Number of opened lanes

11. Number of closed lanes

13. Work zone duration (Short/Long)

15. Work time (Day/ Night)

17. Weekday
2. Lane width

4. Lane closure location (Left/ Right)

6. Work zone length

8. Weather condition (Clear/ Rain)

10. Driver composition and population

12. Ramp (Yes/ No)

14. Work zone speed limit

16. Normal speed

18. Lateral clearance

Table 2.1 shows that heavy vehicle percentage, work zone grade, and work zone intensity are the most common parameters in the previously developed models. However, some variables, such as lateral clearance or weather conditions, are infrequent in the proposed models.

\subsection{WORK ZONES AND ROADWAY DATA}

UDOT stores work zones data as well as traffic data in its databases, and those databases are updated frequently. Since the ongoing projects may keep changing the work zone setups, this project only focused on the completed projects. Using the Google Earth file of completed projects, we can get the locations and project attributes. Figure 3.1 shows a screenshot of the attributes associated with each project. Each project has more than 170 attributes; however, some of them, such as its contractor, project cost, and engineering company, do not affect the traffic conditions of roads. 


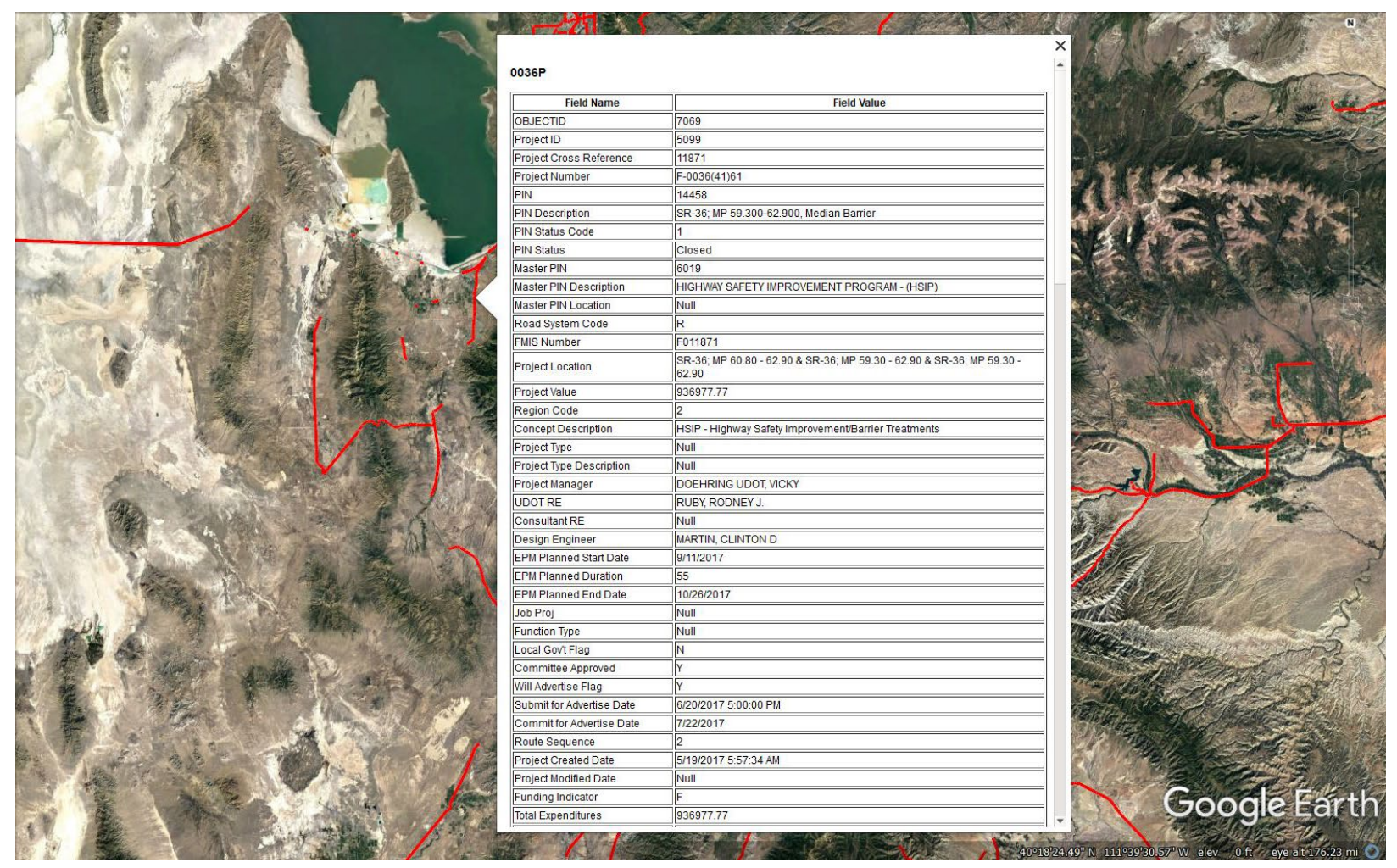

Figure 3.1: A Screenshot of Google Earth Database

In order to increase the readability, Figure 3.2 shows a screenshot of the project features associated with each project. Roadway features, such as grade, heavy vehicle percentage, and lane width, could not be extracted from the work zones database. Utah open data portal provides collected data from statewide roads yearly. Table 3-1 shows a small part of the work zone collected data. 
0036P

\begin{tabular}{|c|c|}
\hline Field Name & Field Value \\
\hline OBJECTID & 7069 \\
\hline Project ID & 5099 \\
\hline Project Cross Reference & 11871 \\
\hline Project Number & $\mathrm{F}-0036(41) 61$ \\
\hline PIN & 14458 \\
\hline PIN Description & SR-36; MP 59.300-62.900, Median Barrier \\
\hline PIN Status Code & 1 \\
\hline PIN Status & Closed \\
\hline Master PIN & 6019 \\
\hline Master PIN Description & HIGHWAY SAFETY IMPROVEMENT PROGRAM - (HSIP) \\
\hline Master PIN Location & Null \\
\hline Road System Code & $\mathrm{R}$ \\
\hline FMIS Number & F011871 \\
\hline Project Location & $\begin{array}{l}\text { SR-36; MP } 60.80 \text { - } 62.90 \text { \& SR-36; MP } 59.30 \text { - } 62.90 \text { \& SR-36; MP } 59.30 \text { - } \\
62.90\end{array}$ \\
\hline Project Value & 936977.77 \\
\hline Region Code & 2 \\
\hline Concept Description & HSIP - Highway Safety Improvement/Barrier Treatments \\
\hline Project Type & Null \\
\hline Project Type Description & Null \\
\hline Project Manager & DOEHRING UDOT, VICKY \\
\hline UDOT RE & RUBY, RODNEY J. \\
\hline Consultant RE & Null \\
\hline Design Engineer & MARTIN, CLINTON D \\
\hline EPM Planned Start Date & 9/11/2017 \\
\hline EPM Planned Duration & 55 \\
\hline EPM Planned End Date & $10 / 26 / 2017$ \\
\hline Job Proj & Null \\
\hline Function Type & Null \\
\hline Local Gov't Flag & $\mathrm{N}$ \\
\hline Committee Approved & $\mathrm{Y}$ \\
\hline Will Advertise Flag & $\mathrm{Y}$ \\
\hline Submit for Advertise Date & 6/20/2017 5:00:00 PM \\
\hline Commit for Advertise Date & $7 / 22 / 2017$ \\
\hline Route Sequence & 2 \\
\hline Project Created Date & 5/19/2017 5:57:34 AM \\
\hline Project Modified Date & Null \\
\hline Funding Indicator & $F$ \\
\hline Total Expenditures & 936977.77 \\
\hline
\end{tabular}

Figure 3.2: Projects Attributes 
Table 3.1: Data Collection Spreadsheet

\begin{tabular}{|c|c|c|c|c|c|c|c|c|}
\hline 1766 & 10219 & $R$ & $0121 P$ & 39.834 & 40.293 & $3 / 15 / 2015$ & 43 & $10 / 11 / 2015$ \\
\hline 1903 & 10006 & $\mathrm{~N}$ & 154 & 5.3 & 6.2 & $3 / 21 / 2013$ & 128 & $11 / 18 / 2013$ \\
\hline 1984 & 8715 & $\mathrm{R}$ & $2552 \mathrm{P}$ & 0.56 & 0.851 & $5 / 5 / 2016$ & 90 & $8 / 13 / 2016$ \\
\hline 2002 & 8813 & NA & $0114 \mathrm{P}$ & 9.029 & 9.029 & $9 / 19 / 2013$ & 30 & $10 / 16 / 2013$ \\
\hline 2264 & 10842 & $\mathrm{~N}$ & 0089P & 37.81 & 46.04 & $8 / 1 / 2018$ & 80 & $7 / 11 / 2018$ \\
\hline 2430 & 10854 & $\mathrm{R}$ & $0290 \mathrm{P}$ & 0 & 1.165 & $5 / 23 / 2013$ & 78 & $7 / 29 / 2013$ \\
\hline 2445 & 10859 & $\mathrm{R}$ & $1327 \mathrm{P}$ & 0.385 & 0.727 & $5 / 2 / 2016$ & 75 & $9 / 8 / 2016$ \\
\hline 2451 & 10890 & u & 0186NR00809 & 0.279 & 0.319 & $8 / 23 / 2017$ & 150 & $3 / 6 / 2018$ \\
\hline 2511 & 10010 & 1 & $0015 \mathrm{~N}$ & 293.63 & 309.33 & $7 / 5 / 2016$ & 80 & $11 / 22 / 2016$ \\
\hline 2548 & 10664 & u & $3158 \mathrm{P}$ & 3.29 & 3.8 & $6 / 3 / 2013$ & 50 & $8 / 14 / 2013$ \\
\hline 2567 & 10585 & 1 & $0015 B$ & 206.493 & 207.062 & $5 / 11 / 2015$ & 30 & $6 / 14 / 2016$ \\
\hline 2930 & 11493 & 1 & $0080 \mathrm{~N}$ & 30 & 41.278 & $5 / 15 / 2017$ & 60 & $11 / 21 / 2017$ \\
\hline 2932 & 11494 & N & $0173 P$ & 7.05 & 9.199 & $9 / 2 / 2014$ & 120 & $10 / 3 / 2014$ \\
\hline 3039 & 10890 & $u$ & 0186PR00806 & 0.319 & 0.361 & $7 / 8 / 2013$ & 150 & $9 / 30 / 2013$ \\
\hline 3040 & 10890 & $u$ & OOBOP & 127.055 & 127.062 & $7 / 8 / 2013$ & 150 & $9 / 30 / 2013$ \\
\hline 3123 & 10890 & $u$ & $2272 \mathrm{P}$ & 0.403 & 0.422 & $10 / 20 / 2015$ & 150 & $11 / 20 / 2015$ \\
\hline 3161 & 10708 & $\mathrm{~N}$ & 0089P & 407.79 & 408.64 & $6 / 12 / 2017$ & 60 & $10 / 10 / 2017$ \\
\hline 3262 & 12161 & $\mathrm{~N}$ & $0063 \mathrm{P}$ & 1.5 & 2.6 & $5 / 1 / 2017$ & 55 & $6 / 16 / 2017$ \\
\hline 3263 & 12161 & $\mathrm{~N}$ & $0063 P$ & 1.5 & 2.6 & $12 / 18 / 2014$ & 55 & $4 / 17 / 2015$ \\
\hline 3448 & 12161 & $N$ & $0063 P$ & 1.5 & 2.6 & $8 / 22 / 2016$ & 55 & $1 / 19 / 2017$ \\
\hline 3517 & 11420 & 1 & OOBOP & 161.454 & 161.495 & $5 / 27 / 2014$ & 150 & $7 / 12 / 2014$ \\
\hline 3556 & 11372 & 1 & $0015 P$ & 0 & 140 & $6 / 5 / 2017$ & 112 & $7 / 29 / 2017$ \\
\hline 3698 & 11367 & U & $0202 \mathrm{P}$ & 0 & 0.57 & $7 / 8 / 2013$ & 120 & $10 / 23 / 2013$ \\
\hline 3709 & 11430 & $R$ & 0020P & 10 & 12 & $5 / 12 / 2014$ & 90 & $8 / 5 / 2014$ \\
\hline 3710 & 11431 & $R$ & 0191P & 86 & 87.5 & $5 / 13 / 2019$ & 65 & $7 / 21 / 2019$ \\
\hline 3764 & 12503 & $R$ & 0163P & 11.015 & 20.899 & $11 / 3 / 2014$ & 76 & $12 / 3 / 2014$ \\
\hline 3766 & 12465 & $R$ & 0130P & 6.507 & 20.25 & $7 / 12 / 2018$ & 75 & $6 / 1 / 2018$ \\
\hline 3771 & 12246 & $u$ & 0052 NR00404 & 0 & 0.092 & $12 / 28 / 2015$ & 49 & $5 / 26 / 2016$ \\
\hline 3783 & 12268 & u & $0013 P$ & 0.63 & 2.53 & $4 / 3 / 2017$ & 30 & $6 / 22 / 2017$ \\
\hline 3805 & 12281 & u & 0106P & 0 & 1.048 & $8 / 20 / 2018$ & 150 & $11 / 22 / 2018$ \\
\hline 3806 & 12281 & u & 0106P & 0 & 1.048 & $8 / 20 / 2018$ & 150 & $11 / 22 / 2018$ \\
\hline 3876 & 12279 & u & 0232P & 0.45 & 2.25 & $4 / 13 / 2015$ & 192 & $9 / 24 / 2015$ \\
\hline 3974 & 12298 & 1 & $0015 P$ & 365.508 & 373.26 & $6 / 1 / 2016$ & 60 & $12 / 8 / 2016$ \\
\hline 4034 & 12205 & $\mathrm{R}$ & 0143P & 9.4 & 10.2 & $6 / 20 / 2016$ & 273 & $8 / 23 / 2016$ \\
\hline 4043 & 12235 & $R$ & $0191 P$ & 0 & 12.6 & $12 / 28 / 2015$ & 55 & $5 / 26 / 2016$ \\
\hline 4442 & 12631 & U & 0037P & 1.502 & 1.502 & $6 / 6 / 2016$ & 45 & $8 / 8 / 2016$ \\
\hline 4538 & 12363 & N & 0036P & 56.78 & 62.655 & $7 / 6 / 2015$ & 40 & $6 / 20 / 2016$ \\
\hline 4552 & 12985 & I & $0015 \mathrm{~N}$ & 280.2 & 283.3 & $5 / 1 / 2018$ & 25 & $7 / 31 / 2018$ \\
\hline 4688 & 12638 & U & $0111 \mathrm{P}$ & 9.105 & 10.09 & $7 / 26 / 2016$ & 90 & $9 / 20 / 2016$ \\
\hline 4792 & 13458 & $u$ & 0209p & 15.89 & 18.84 & $4 / 24 / 2017$ & 60 & $9 / 6 / 2017$ \\
\hline
\end{tabular}


Table 3.2 cont. : Data Collection Spreadsheet

\begin{tabular}{|c|c|c|c|c|c|c|c|c|}
\hline PROJ LOC $>$ & REG v & CONCEPT DESC $>$ & PROI TYP NM - & LENGTH & Lane W - & Grade - & Heavy Vehil - & Heavy Vehic - \\
\hline SR-121; MP .00 & 3 & Minor Rehabilitatiol & Roadway & 0.459 & 12 & 0 & 0.229 & 0.092 \\
\hline State Route: SF & & Intersection Improve & ETraffic and Safety & 0.9 & 12 & 0.444444 & 0.08533 & 0.05339 \\
\hline Cnty:FA-2552; N & & RECONSTRUCTION & Roadway Work & 0.291 & - & - & - & - \\
\hline SR-114; MP 9.0 E & & Railway-Highway Gr; & Traffic and Safety & 0 & 12 & 1 & - & - \\
\hline US-89; MP 37.8: & & Preservation - Roadv & Roadway & 8.23 & 12 & 1.369048 & 0.084 & 0.127 \\
\hline SR-290; MP .00 & 4 & Major Rehabilitatior & Roadway & 1.165 & 11 & 1.399141 & 0.089 & 0.049 \\
\hline 1900 East Price & & Small Urban & Joint Highway Corr & 0.342 & - & - & - & - \\
\hline TO UTILITY; MP & & Replacement and $\mathrm{Re}$ & E Structures & 0.04 & 12 & - & 0.041 & 0.029 \\
\hline I-15; MP 293.63 & & Preservation High Vc & CPreservation & 15.7 & 11 & 0.874068 & 0.09369414 & 0.051651911 \\
\hline Cnty:FA-3158; N & & Reconstruct widenin & Roadway & 0.51 & - & - & - & - \\
\hline I-15; MP 206.49 & & Preservation - Struct & Structures & 0.569 & 12 & - & 0.02 & 0.2 \\
\hline 1-80; MP $30.00-$ & & Minor Rehabilitatiol & I Roadway & 11.278 & 12 & 0.035467 & 0.068 & 0.443 \\
\hline SR-173; MP 7.05 & & Minor Rehabilitatiol & Roadway & 2.149 & 10 & 1.226364 & 0.057978 & 0.01742 \\
\hline TO UTILITY; MP & & Replacement and Re & E Structures & 0.042 & 12 & 5 & 0.041 & 0.029 \\
\hline TO UTILITY; MP & & Replacement and Re & E Structures & 0.007 & 12 & 2 & 0.062 & 0.072 \\
\hline TO UTILITY; MP & & Replacement and $\mathrm{Re}$ & E Structures & 0.019 & - & - & - & - \\
\hline US-89; MP 407.: & & Choke Point & Capacity & 0.85 & 11 & 1 & 0.073533 & 0.02652 \\
\hline SR-12; MP $10.7 \mathrm{C}$ & & Pedestrian/Bicycle S & Traffic and Safety & 1.1 & 11 & 0.363636 & 0.114 & 0.028 \\
\hline SR-12; MP $10.7 \mathrm{C}$ & & Pedestrian/Bicycle S & Traffic and Safety & 1.1 & 11 & 0.363636 & 0.114 & 0.028 \\
\hline SR-12; MP $10.7 \mathrm{C}$ & & Pedestrian/Bicycle S & Traffic and Safety & 1.1 & 11 & 0.363636 & 0.114 & 0.028 \\
\hline 1-80; MP 158.62 & & Replacement and Re & E Structures & 0.041 & 12 & 1 & 0.086 & 0.378 \\
\hline I-15; MP .00-14 & & Barrier - $\mathrm{T} / \mathrm{S}$ & Traffic and Safety & 140 & 12 & 1.423779 & 0.11545295 & 0.220140807 \\
\hline SR-202; MP .00 & 2 & Intersection Improve & ETraffic and Safety & 0.57 & 11 & 0.526316 & 0.268 & 0.083 \\
\hline SR-20; MP $10.0 C$ & & Adding a lane/shoul & Roadway & 2 & 12 & 7.3015 & 0.111 & 0.195 \\
\hline US-191; MP 86.C & & Adding a lane/shou & Roadway & 1.5 & 9 & 2.266667 & 0.105 & 0.278 \\
\hline US-163; MP $11 .($ & & Minor Rehabilitatiol & I Roadway & 9.884 & 11 & 3.232323 & 0.246 & 0.028 \\
\hline SR-130; MP 6.51 & & Preservation - Roadv & Roadway & 13.743 & 11 & 0.652174 & 0.103461 & 0.127005 \\
\hline SR-52; MP 1.54 & 3 & Preservation High Vc & c Preservation & 0.092 & 14 & - & 0.163 & 0.038 \\
\hline SR-13; MP .63 - & 1 & Preservation - Roadv & Roadway & 1.9 & 10 & 2 & 0.107908 & 0.066098 \\
\hline SR-106; MP .00 & 1 & Major Rehabilitatior & I Roadway & 1.048 & 10 & 2.225455 & 0.043 & 0.064 \\
\hline SR-106; MP .00 & 1 & Major Rehabilitatior & Roadway & 1.048 & 10 & 2.225455 & 0.043 & 0.064 \\
\hline SR-232; MP .45 & 1 & Minor Rehabilitatiol & Roadway & 1.8 & 11 & 3.612778 & 0.050637 & 0.041542 \\
\hline I-15; MP 365.51 & 11 & Rehabilitation High & Rehabilitation & 7.752 & 12 & 0.116099 & 0.227743034 & 0.287895253 \\
\hline SR-143; MP 9.4C & & Spot Safety/Barrier - & Traffic and Safety & 0.8 & 11 & 7.5 & 0.087 & 0.059 \\
\hline US-191; MP 103 & & Preservation - Roadv & Roadway & 12.6 & 10 & 0.975806 & 0.12 & 0.15 \\
\hline SR-97; MP 4.09 & 1 & ITS Communications & Traffic Manageme & 0 & 12 & 1 & - & - \\
\hline SR-36; MP 55.77 & & Preservation - Roadv & Roadway & 5.875 & 12 & 1.804255 & 0.179 & 0.046 \\
\hline I-15; MP 280.20 & & Highway Safety Impr & Traffic and Safety & 3.1 & 10 & 1.073171 & 0.114019032 & 0.089951613 \\
\hline Cnty:FA-2061; N & & ITS Communications & Traffic Manageme & 0.985 & 11 & 2.7 & 0.186 & 0.091 \\
\hline SR-209; MP $15 . \varepsilon$ & & Minor Rehabilitatiol & I Roadway & 2.95 & 10 & 4.33121 & 0.055 & 0.032 \\
\hline
\end{tabular}


Table 3.3 cont. : Data Collection Spreadsheet

\begin{tabular}{|c|c|c|c|c|}
\hline Norme - & ROUTE CLASS - & START DAT - DAYS - & SUBSTANTIALLY $\mathrm{d}-$ CNTY NAME - & CNTY CD $\vee$ \\
\hline 35 & STATE & $7 / 7 / 201443$ & $10 / 29 / 2014$ VARIOUS & 97 \\
\hline 60 & STATE & $2 / 1 / 2013128$ & $11 / 19 / 2013$ SALT LAKE & 35 \\
\hline- & COUNTY & $5 / 15 / 201380$ & $8 / 8 / 2013$ SEVIER & 41 \\
\hline 50 & STATE & $9 / 19 / 201330$ & $10 / 16 / 2013$ UTAH & 49 \\
\hline 65 & STATE & $8 / 5 / 201351$ & $10 / 21 / 2013$ KANE & 25 \\
\hline- & STATE & $6 / 3 / 201375$ & $8 / 28 / 2013$ SANPETE & 39 \\
\hline - & COUNTY & $4 / 3 / 201870$ & $9 / 18 / 2018$ CARBON & 7 \\
\hline - & STATE & $5 / 8 / 2017150$ & 3/8/2018 SALT LAKE & 35 \\
\hline 70 & STATE & $1 / 14 / 201886$ & $6 / 13 / 2018$ SALT LAKE & 35 \\
\hline - & COUNTY & $3 / 28 / 201650$ & 5/11/2016 WASHINGTON & 53 \\
\hline 80 & STATE & $6 / 4 / 201221$ & $7 / 4 / 2012$ JUAB & 23 \\
\hline 80 & STATE & $4 / 18 / 201660$ & $6 / 17 / 2016$ TOOELE & 45 \\
\hline 45 & STATE & $6 / 15 / 2015120$ & 10/13/2015 SALT LAKE & 35 \\
\hline 50 & STATE & $5 / 8 / 2017150$ & 3/8/2018 SALT LAKE & 35 \\
\hline 65 & STATE & $5 / 8 / 2017150$ & $3 / 8 / 2018$ SALT LAKE & 35 \\
\hline- & COUNTY & $5 / 8 / 2017150$ & $3 / 8 / 2018$ SALT LAKE & 35 \\
\hline 55 & STATE & $5 / 26 / 2015115$ & $12 / 13 / 2016$ WEBER & 57 \\
\hline 55 & STATE & $8 / 27 / 201555$ & 10/24/2015 ROUTE ERROR & 90 \\
\hline 55 & STATE & $8 / 27 / 201555$ & 10/24/2015 ROUTE ERROR & 90 \\
\hline 55 & STATE & $8 / 27 / 201555$ & 10/24/2015 ROUTE ERROR & 90 \\
\hline 70 & STATE & $6 / 1 / 2015150$ & 4/9/2016 SUMMIT & 43 \\
\hline 75 & STATE & $11 / 18 / 2013112$ & $11 / 23 / 2014$ VARIOUS & 97 \\
\hline 45 & STATE & $12 / 18 / 2014120$ & 10/18/2015 SALT LAKE & 35 \\
\hline 40 & STATE & $4 / 25 / 2016251$ & $10 / 14 / 2016$ IRON & 21 \\
\hline 55 & STATE & $9 / 12 / 201652$ & $10 / 28 / 2016$ SAN JUAN & 37 \\
\hline 60 & STATE & $8 / 15 / 201676$ & $10 / 28 / 2016$ SAN JUAN & 37 \\
\hline 60 & STATE & $8 / 11 / 201675$ & 10/14/2016 IRON & 21 \\
\hline- & STATE & $6 / 6 / 201849$ & $9 / 21 / 2018$ UTAH & 49 \\
\hline 35 & STATE & $5 / 21 / 201628$ & 6/19/2016 BOX ELDER & 3 \\
\hline 35 & STATE & $1 / 14 / 2016220$ & $6 / 7 / 2017$ DAVIS & 11 \\
\hline 35 & STATE & $1 / 14 / 2016220$ & $6 / 7 / 2017$ DAVIS & 11 \\
\hline 40 & STATE & $12 / 28 / 2015150$ & $5 / 22 / 2017$ DAVIS & 11 \\
\hline 75 & STATE & $2 / 15 / 201656$ & 2/1/2018 BOX ELDER & 3 \\
\hline 40 & STATE & $2 / 17 / 201780$ & $5 / 17 / 2018$ IRON & 21 \\
\hline 65 & STATE & $9 / 29 / 201455$ & $11 / 13 / 2014$ SAN JUAN & 37 \\
\hline 40 & STATE & $8 / 14 / 201790$ & $12 / 18 / 2017$ VARIOUS & 97 \\
\hline 55 & STATE & $8 / 19 / 201540$ & 10/28/2015 TOOELE & 45 \\
\hline 70 & STATE & $9 / 30 / 201525$ & 10/16/2015 UTAH & 49 \\
\hline- & STATE & $9 / 17 / 201885$ & 2/19/2019 SALT LAKE & 35 \\
\hline 40 & STATE & $6 / 5 / 201785$ & $8 / 29 / 2017$ SALT LAKE & 35 \\
\hline
\end{tabular}




\subsection{TRAFFIC DATA}

Using the location of work zones and UDOT's traffic management platform, we can find the adjacent traffic sensors to each work zone. Figure 3.3 shows the location of traffic sensors on state roadways.

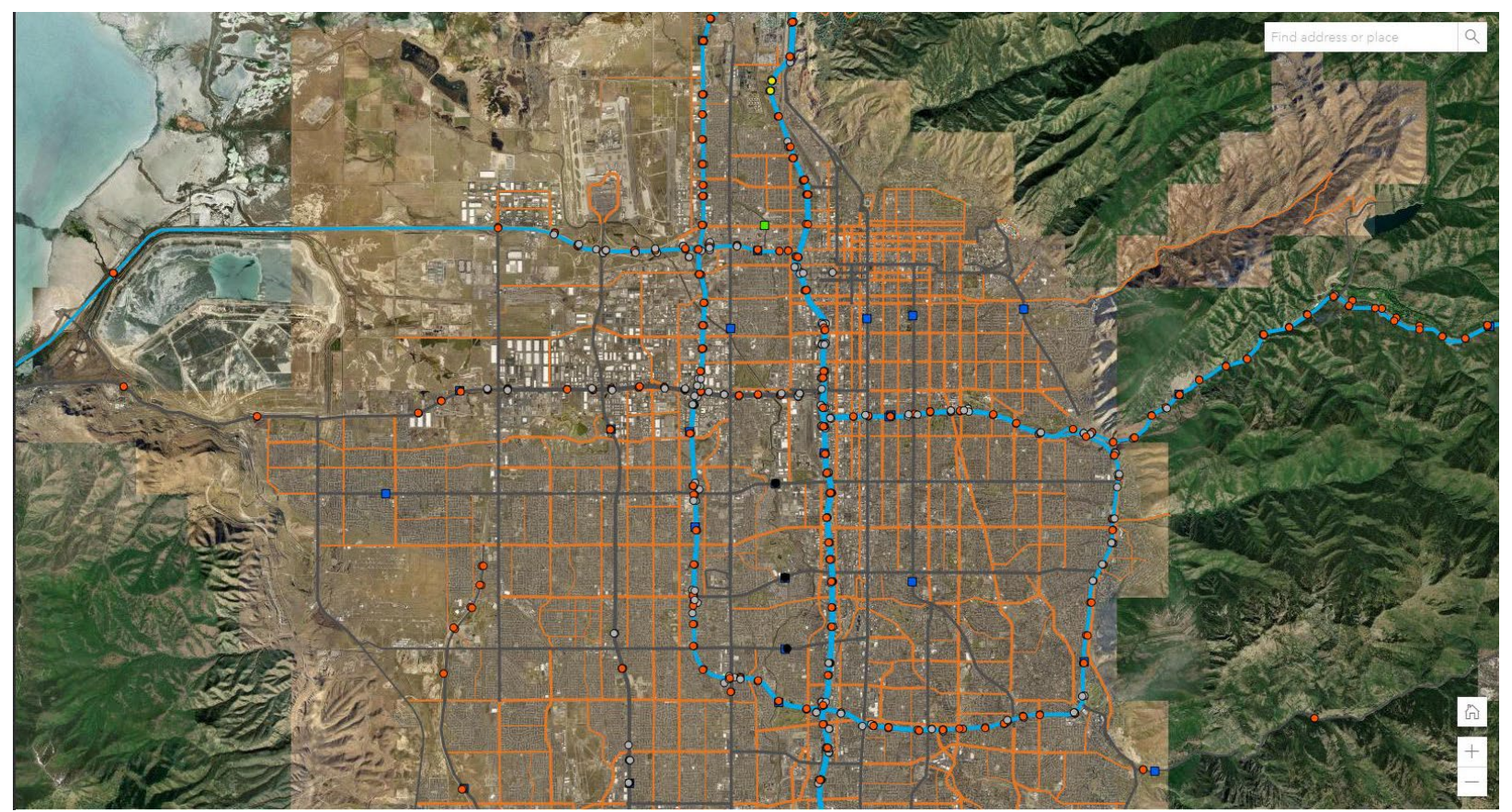

Figure 3.3: Location of Sensors on the Roadways of the State

Using the sensors' IDs and the occurrence date of work zones, we can extract the traffic data related to each work zone. Hourly traffic data during the project duration was used for training the model. Table 3.4 shows the location of work zones and the adjacent sensor ID used to extract traffic data. Furthermore, Table 3.5 shows the extracted traffic data for a specific location using the PeMS database.

After collecting data from different data sources, one crucial step is to preprocess data. In any data-driven process, data preprocessing is the step of transforming and encoding data in order to bring it to such a state that the machine can parse it. Especially, the features of the data can now be interpreted by the algorithm. 
Table 3.4: Work zones Location and the Adjacent Sensors

\begin{tabular}{|c|c|c|c|c|c|c|c|}
\hline LOCATION & $\mathbf{Y}$ & $\mathrm{x}$ & Sensors \# & ID & Road & Sensor $X$ & Sensory \\
\hline ['SR-201;', 'MP', 5.83, '-', 10.93, '\&', 'SR-201;', 'MP', 5.82, '-', 10.92] & 40.72571093 & -112.022447 & 100353 & 300 & SR-201 & -112.02891 & 40.7245 \\
\hline$[' I-80 ; '$, 'MP', 136, 'to', 143] & 40.74002853 & -111.674719 & 100429 & 618 & $1-80$ & -111.65675 & 40.7425 \\
\hline ['I-80;', 'MP', 136, 'to', 143] & 40.75215523 & -111.61349 & 100171 & 622 & $1-80$ & -111.61488 & 40.7525 \\
\hline ['SR-201;', 'MP', 5.83, '-', 10.93, '\&', 'SR-201;', 'MP', 5.82, '-', 10.92] & 40.72591886 & -112.021702 & 100353 & 300 & SR-201 & -112.02891 & 40.7245 \\
\hline ['I-80;', 'MP', 133.6, '-', 136.3, '\&', 'I-80;', 'MP', 133.6, '-', 136.3] & 40.75201512 & -111.715958 & 100618 & 611 & $1-80$ & -111.72449 & 40.7463 \\
\hline ['I-80;', 'MP', 133.6, '-', 136.3, '\&', 'I-80;', 'MP', 133.6, '-', 136.3] & 40.73925033 & -111.669219 & 100429 & 618 & $1-80$ & -111.65675 & 40.7425 \\
\hline ['I-15;', 'ITS', 'PROJECTS', 'AT', 'VARIOUS', 'LOCATIONS'] & 36.99999037 & -113.62243 & 100210 & 790 & $1-15$ & -113.60886 & 37.0214 \\
\hline ['I-15;', 'MP', 293.63, '-', 309.33, '\&', 'I-15;', 'MP', 293.63, '-', 309.33] & 40.55888897 & -111.898131 & 100035 & 570 & $1-15$ & -111.8988 & 40.5583 \\
\hline ['I-15;', 'MP', 293.63, '-', 309.33, '\&', 'I-15;', 'MP', 293.63, '-', 309.33] & 40.78237808 & -111.910585 & 100615 & 436 & $1-15$ & -111.91034 & 40.7717 \\
\hline ['I-15;', 'MP', 334.73, 'to', 'MP', 340.74] & 41.11121916 & -112.010814 & 100340 & 978 & $1-15$ & -112.01526 & 41.1145 \\
\hline ['I-15;', 'MP', 334.73, 'to', 'MP', 340.74] & 41.19089758 & -112.005484 & 100128 & 966 & $1-15$ & -112.00663 & 41.1916 \\
\hline ['US-6;', 'MP', 176.91, '-', 177.6] & 40.09268015 & -111.600023 & 100364 & $3 E+06$ & US-6 & -111.59892 & 40.0916 \\
\hline ['I-15;', 'MP', 303.34, '-', 303.94, '\&', 'SR-171;', 'MP', 9.23, '-', 10.27] & 40.69836923 & -111.901627 & 100566 & 530 & $1-15$ & -111.90428 & 40.6967 \\
\hline ['I-15;', 'MP', 303.34, '-', 303.94, '\&', 'SR-171;', 'MP', 9.23, '-', 10.27] & 40.70700917 & -111.90357 & 100554 & 526 & $1-15$ & -111.9044 & 40.7086 \\
\hline ['I-15;', 'MP', 293.63, '-', 309.33, '\&', 'I-15;', 'MP', 293.63, '-', 309.33] & 40.55888897 & -111.898131 & 100035 & 570 & $1-15$ & -111.8988 & 40.5583 \\
\hline ['I-15;', 'MP', 293.63, '-', 309.33, '\&', 'I-15;', 'MP', 293.63, '-', 309.33] & 40.78237808 & -111.910585 & 100615 & 436 & $1-15$ & -111.91034 & 40.7717 \\
\hline ['I-15;', 'MP', 279.8, '-', 291.5] & 40.38931982 & -111.833926 & 100403 & $3 E+06$ & $1-15$ & -111.83554 & 40.3916 \\
\hline ['I-15;', 'MP', 279.8, '-', 291.5] & 40.5287961 & -111.891015 & 100053 & 375 & $1-15$ & -111.8921 & 40.5354 \\
\hline ['I-15;', 'MP', 361.5, '-', 362.5] & 41.47817213 & -112.053873 & 100593 & $3 E+06$ & $1-15$ & -112.05609 & 41.4646 \\
\hline ['I-15;', 'MP', 361.5, '-', 362.5] & 41.49255703 & -112.053943 & 100593 & $3 E+06$ & $1-15$ & -112.05609 & 41.4646 \\
\hline ['TO', 'UTILITY;', 'MP', 0.19, '-', 0.2, '\&', 'RANCH', 'EXIT', 'OVERPASS;', 'Ml & 40.71223778 & -111.789757 & 100607 & 192 & $1-215$ & -111.79618 & 40.708 \\
\hline ['TO', 'UTILITY;', 'MP', 0.19, '-', 0.2, '\&', 'RANCH', 'EXIT', 'OVERPASS;', 'Ml & 40.71194586 & -111.78992 & 100607 & 192 & $1-215$ & -111.79618 & 40.708 \\
\hline ['TO', 'UTILITY;', 'MP', 0.19, '-', 0.2, '\&', 'RANCH', 'EXIT', 'OVERPASS;', 'Ml & 40.7073884 & -111.796871 & 100356 & 192 & $1-215$ & -111.79725 & 40.708 \\
\hline ['TO', 'UTILITY;', 'MP', 0.19 , '-', 0.2, '\&', 'RANCH', 'EXIT', 'OVERPASS;', 'M & 40.70715268 & -111.797022 & 100356 & 192 & $1-215$ & -111.79725 & 40.708 \\
\hline ['I-15;', 'MP', 331.53, '-', 334.73, '\&', 'I-15;', 'MP', 342.24, '-', 344.57] & 41.20913737 & -111.996331 & 100124 & 922 & $1-15$ & -111.99861 & 41.2128 \\
\hline ['I-15;', 'MP', 331.53, '-', 334.73, '\&', 'I-15;', 'MP', 342.24, '-', 344.57] & 41.23873271 & -112.014458 & 100136 & 954 & $1-15$ & -112.01297 & 41.2284 \\
\hline ['I-215;', 0.736, 'to', 1.707] & 40.70154961 & -111.795065 & 100604 & 190 & $1-215$ & -111.79476 & 40.7015 \\
\hline$[' I-215 ; ', 0.736$, 'to', 1.707$]$ & 40.71218179 & -111.805847 & & & & & \\
\hline ['I-15;', 'MP', 331.53, '-', 334.73, '\&', 'I-15;', 'MP', 331.53, '-', 334.73] & 41.07301764 & -111.976065 & 100375 & 738 & $1-15$ & -111.9624 & 41.0522 \\
\hline ['I-15;', 'MP', 331.53, '-', 334.73, '\&', 'I-15;', 'MP', 331.53, '-', 334.73] & 41.11107441 & -112.0111 & 100340 & 978 & $1-15$ & -112.01526 & 41.1145 \\
\hline ['I-15;', 'MP', 331.53, '-', 334.73, '\&', 'I-15;', 'MP', 331.53, '-', 334.73] & 41.07306568 & -111.975774 & 100375 & 738 & $1-15$ & -111.9624 & 41.0522 \\
\hline ['I-15;', 'MP', 331.53, '-', 334.73, '\&', 'I-15;', 'MP', 331.53, '-', 334.73] & 41.11121916 & -112.010814 & 100340 & 978 & $1-15$ & -112.01526 & 41.1145 \\
\hline ['US-189;', 'MP', 7.43, '-', 24.9] & 40.31977962 & -111.649365 & 100228 & 468 & US-189 & -111.64222 & 40.3233 \\
\hline ['US-189;', 'MP', 7.43, '-', 24.9] & 40.40870975 & -111.500903 & 100237 & 475 & US-189 & -111.53608 & 40.3996 \\
\hline ['I-15;', 'MP', 10.88, '-', 10.97, '\&', 'SR-9;', 'MP', 3.58, '-', 3.71, '\&', 'I-15;', & 37.12813535 & -113.525831 & 100211 & 795 & $1-15$ & -113.54773 & 37.1172 \\
\hline ['I-15;', 'MP', 10.88, '-', 10.97, '\&', 'SR-9;', 'MP', 3.58, '-', 3.71, '\&', 'I-15;', & 37.12917376 & -113.524242 & 100211 & 795 & $1-15$ & -113.54773 & 37.1172 \\
\hline ['FROM', 'I-215N;', 'MP', 0, '-', 1.25, '\&', 'FROM', 'I-215N', 'TO', 'I-80P', '\& & 40.7404851 & -111.949261 & 100158 & 73 & $1-215$ & -111.94737 & 40.75 \\
\hline ['FROM', 'I-215N;', 'MP', 0, '-', 1.25, '\&', 'FROM', 'I-215N', 'TO', 'I-80P', '\& & 40.73995035 & -111.949744 & 100158 & 73 & $1-215$ & -111.94737 & 40.75 \\
\hline ['FROM', 'I-215N;', 'MP', 0, '-', 1.25, '\&', 'FROM', 'I-215N', 'TO', 'I-80P', '8 & 40.76168102 & -111.949793 & 100551 & 74 & $1-215$ & -111.95039 & 40.7587 \\
\hline ['FROM', 'I-215N;', 'MP', 0, '-', 1.25, '\&', 'FROM', 'I-215N', 'TO', 'I-80P', '\& & 40.76594218 & -111.956316 & 100368 & 207 & $1-80$ & -111.9541 & 40.7627 \\
\hline ['FROM', 'I-215N;', 'MP', 0, '-', 1.25, '\&', 'FROM', 'I-215N', 'TO', 'I-80P', '\& & 40.76497491 & -111.957048 & 100368 & 207 & $1-80$ & -111.9541 & 40.7627 \\
\hline ['FROM', 'I-215N;', 'MP', 0, '-', 1.25, '\&', 'FROM', 'I-215N', 'TO', 'I-80P', '\& & 40.76541596 & -111.939121 & 100341 & 214 & $1-80$ & -111.9297 & 40.7642 \\
\hline ['FROM', 'I-215N;', 'MP', 0, '-', 1.25, '\&', 'FROM', 'I-215N', 'TO', 'I-80P', '\& & 40.76649459 & -111.946568 & 100368 & 207 & $1-80$ & -111.9541 & 40.7627 \\
\hline ['FROM', 'I-215N;', 'MP', 0, '-', 1.25, '\&', 'FROM', 'I-215N', 'TO', 'I-80P', '\& & 40.76525266 & -111.956143 & 100368 & 207 & $1-80$ & -111.9541 & 40.7627 \\
\hline ['I-15;', 'MP', 325.93, '-', 329.85, '\&', 'I-15;', 'MP', 325.93, '-', 329.85] & 41.0031366 & -111.927573 & 100254 & 907 & $1-15$ & -111.93044 & 41.0066 \\
\hline ['I-15;', 'MP', 325.93, '-', 329.85, '\&', 'I-15;', 'MP', 325.93, '-', 329.85] & 41.05253847 & -111.96061 & 100375 & 738 & $1-15$ & 624 & 41.0522 \\
\hline ['I-80;', 'MP', 127.57, '-', 139.22, '\&', 'I-80;', 'MP', 127.57, '-', 139.22] & 40.71444991 & -111.808598 & 100390 & 193 & $1-80$ & -111.8061 & 40.7128 \\
\hline
\end{tabular}


Table 3.5: Extracted Traffic Data using the PeMS Database

\begin{tabular}{|c|c|c|c|c|}
\hline & & & \multicolumn{2}{|c|}{ Data Quality } \\
\hline Hour & Lane 1 Flow (Veh/Hour) & Lane 2 Flow (Veh/Hour) & \# Lane Points & $\%$ Observed \\
\hline $05 / 05 / 202100: 00$ & 24.0 & $\begin{array}{r}93.0 \\
\end{array}$ & 36 & 100.0 \\
\hline $05 / 05 / 202101: 00$ & 19.0 & 85.0 & 36 & 75.0 \\
\hline $05 / 05 / 202102: 00$ & 21.0 & 82.0 & 36 & 88.9 \\
\hline 05/05/2021 03:00 & 38.0 & 108.0 & 36 & 100.0 \\
\hline $05 / 05 / 202104: 00$ & 68.0 & 277.0 & 36 & 100.0 \\
\hline $05 / 05 / 202105: 00$ & 345.0 & 681.0 & 36 & 100.0 \\
\hline $05 / 05 / 202106: 00$ & 946.0 & $1,180.0$ & 36 & 100.0 \\
\hline $05 / 05 / 202107: 00$ & $1,241.0$ & $1,410.0$ & 36 & 100.0 \\
\hline 05/05/2021 08:00 & 980.0 & $1,219.0$ & 36 & 100.0 \\
\hline $05 / 05 / 202109: 00$ & 517.0 & 884.0 & 36 & 100.0 \\
\hline $05 / 05 / 202110: 00$ & 333.0 & 736.0 & 36 & 100.0 \\
\hline $05 / 05 / 202111: 00$ & 354.0 & 798.0 & 36 & 100.0 \\
\hline $05 / 05 / 202112: 00$ & 379.0 & 864.0 & 36 & 100.0 \\
\hline 05/05/2021 13:00 & 454.0 & 838.0 & 36 & 100.0 \\
\hline $05 / 05 / 202114: 00$ & 540.0 & 964.0 & 36 & 33.3 \\
\hline $05 / 05 / 202115: 00$ & 799.0 & $1,132.0$ & 36 & 0.0 \\
\hline $05 / 05 / 202116: 00$ & 965.0 & $1,242.0$ & 36 & 75.0 \\
\hline $05 / 05 / 202117: 00$ & $1,148.0$ & $1,254.0$ & 36 & 100.0 \\
\hline $05 / 05 / 202118: 00$ & 573.0 & 891.0 & 36 & 100.0 \\
\hline $05 / 05 / 202119: 00$ & 332.0 & 670.0 & 36 & 100.0 \\
\hline $05 / 05 / 202120: 00$ & 255.0 & 641.0 & 36 & 100.0 \\
\hline $05 / 05 / 202121: 00$ & 171.0 & 463.0 & 36 & 100.0 \\
\hline $05 / 05 / 202122: 00$ & 113.0 & 351.0 & 36 & 100.0 \\
\hline $05 / 05 / 202123: 00$ & 41.0 & 203.0 & 36 & 100.0 \\
\hline $05 / 06 / 202100: 00$ & 25.0 & 113.0 & 36 & 100.0 \\
\hline $05 / 06 / 202101: 00$ & 15.0 & 64.0 & 36 & 97.2 \\
\hline $05 / 06 / 202102: 00$ & 18.0 & 73.0 & 36 & 97.2 \\
\hline $05 / 06 / 202103: 00$ & 35.0 & 127.0 & 36 & 100.0 \\
\hline $05 / 06 / 202104: 00$ & 78.0 & 244.0 & 36 & 100.0 \\
\hline 05/06/2021 05:00 & 332.0 & 703.0 & 36 & 100.0 \\
\hline $05 / 06 / 202106: 00$ & 892.0 & $1,182.0$ & 36 & 100.0 \\
\hline $05 / 06 / 202107: 00$ & $1,248.0$ & $1,386.0$ & 36 & 100.0 \\
\hline $05 / 06 / 202108: 00$ & 923.0 & $1,215.0$ & 36 & 100.0 \\
\hline 05/06/2021 09:00 & 523.0 & 905.0 & 36 & 100.0 \\
\hline 05/06/2021 10:00 & 395.0 & 824.0 & 36 & 100.0 \\
\hline $05 / 06 / 202111: 00$ & 369.0 & 805.0 & 36 & 100.0 \\
\hline $05 / 06 / 202112: 00$ & 393.0 & 839.0 & 36 & 100.0 \\
\hline $05 / 06 / 202113: 00$ & 455.0 & 892.0 & 36 & 100.0 \\
\hline $05 / 06 / 202114: 00$ & 599.0 & 993.0 & 36 & 100.0 \\
\hline 05/06/2021 15:00 & 843.0 & $1,183.0$ & 36 & 100.0 \\
\hline $05 / 06 / 202116: 00$ & $1,049.0$ & $1,281.0$ & 36 & 100.0 \\
\hline $05 / 06 / 202117: 00$ & $1,212.0$ & $1,391.0$ & 36 & 100.0 \\
\hline $05 / 06 / 202118: 00$ & 677.0 & $1,003.0$ & 36 & 100.0 \\
\hline
\end{tabular}




\subsection{METHODOLOGY}

Many studies have adopted ANN for traffic flow prediction. The robustness of ANN, the capability to handle large datasets, and the parallel processing ability are why it was adopted to estimate historical hourly volumes in the work zones. This section provides a brief overview of the suggested methodology, which would enable researchers and engineers to replicate the analysis. In this section, we first introduce the basic concepts of neural networks. Then, the role of used layers and different evaluation methods for regression models are explained.

\subsection{BASIC CONCEPTS}

The concept of neural networks is adopted from bionomics. As the core components of the brain and the nerve system, neurons form a neural network by interconnecting to billions of other neurons. The outstanding information processing ability of the human brain provides neural networks with the ability to exploit the massively parallel local processing and distribute storage properties in the brain.

The significant difference of human and the computer is in the ability of pattern recognition and learning. Although computers can handle a large number of datasets with high speed, they are not able to distinguish the difference between them. Daily brain tasks such as classification, comparison, learning, and regression are some of the limitations of computers compared to human brains.

ANN is a mathematical system that is able to model the function of a neural network using many of the simple neurons. A neuron converts single or multiple inputs to a single or multiple outputs. The process of converting input values to output is presented in Figure 4.1. The weights assigned to each vector are then updated using the loss value and the backpropagation technique. This technique is the most prevalent method of self-learning for ANN models. In this process, the interconnection weights are adjusted using the error convergence method to obtain a desired output for a given input. The error function at the output neuron is defined as:

$$
E=\frac{1}{2} \sum_{n}\left(T_{j}-A_{j}\right)^{2}
$$

Where $T_{j}$ is the actual value of output neuron, $A_{j}$ is the predicted value of the output neuron, ad $\mathrm{j}$ is the output neuron. The ability to learn, operating in parallel, distributed memory, and fault tolerance are the characteristics of ANN that made them an appropriate choice for various areas. Generally, neural networks are made of three main layers: 1) Input layer, 2) Hidden layer, and 3) Output layer (Figure 4.2).

A fully connected feedforward multilayer neural network, as an example of ANN, consists of multiple layers with neurons in each layer. The first layer represents the 
input layer, the last layer is called the output layer, which is the model prediction, and the layers in between are hidden layers. Each neuron in a fully connected feedforward multilayer neural network is linked with all the neurons from the previous layer. Therefore, the output from a neuron is computed as

$$
a_{i}^{l+1}=f\left(w_{i}^{l+1} a^{l}+b_{i}^{l+1}\right),
$$

Where $a_{i}^{l+1}$ is the output of the $\mathrm{i}$-th neuron in layer $l+1, w_{i}^{l+1}$ is the weight vectors between the i-th neuron in layer $l+1$ and all the neurons in layer $l, a^{l}$ is the output vector from the neurons in layer $l, b_{i}^{l+1}$ is the bias value associated with the $i$-th neuron in layer $l+1$, and $f$ is the activation function used for adding nonlinearity to the model.

Networks with many hidden layers are called deep networks. Such networks are capable of capturing more complex nonlinear relationships. However, overfitting and computational costs are the most common problems of deep neural networks.

Overfitting usually occurs when the proposed model is excessively complex, and the learned pattern from the training dataset is unable to be applied to other datasets. Moreover, increasing the complexity of the model will also result in more parameters that increase the need for more computational resources. Computations are performed with a graphical processing unit (GPU) that can significantly speed up computations. The computations were performed on Nvidia GeForce RTX 2080 Super, using CUDA and PyTorch libraries. The Nvidia GeForce RTX 2080 Super is powerful hardware with 3,072 CUDA cores, and 8GB of GDDR6 clocked at $15.5 \mathrm{Gbps}$. CUDA is a parallel computing platform and programming model developed by Nvidia for general computing on its GPUs. CUDA allows developers to speed up compute-intensive applications by harnessing the power of GPUs for the parallelizable part of the computation [55]. In order to improve the performance of neural networks, various layers have been used in addition to fully connected layers. The following will explain the role of each layer used in the model performance. 


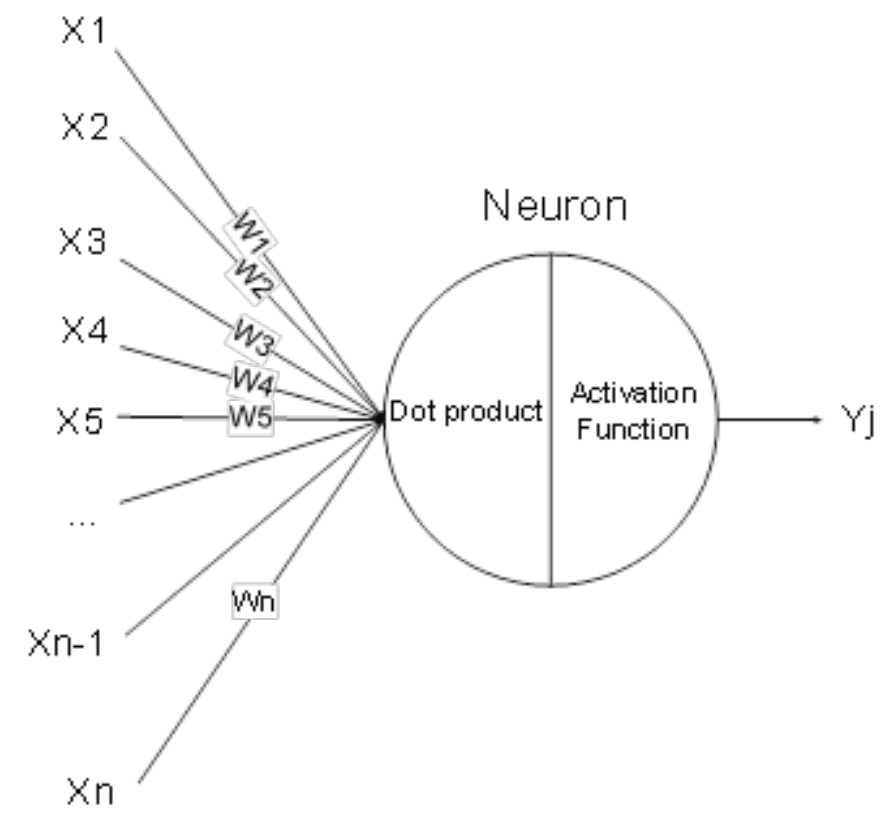

Figure 4.1: An artificial neuron

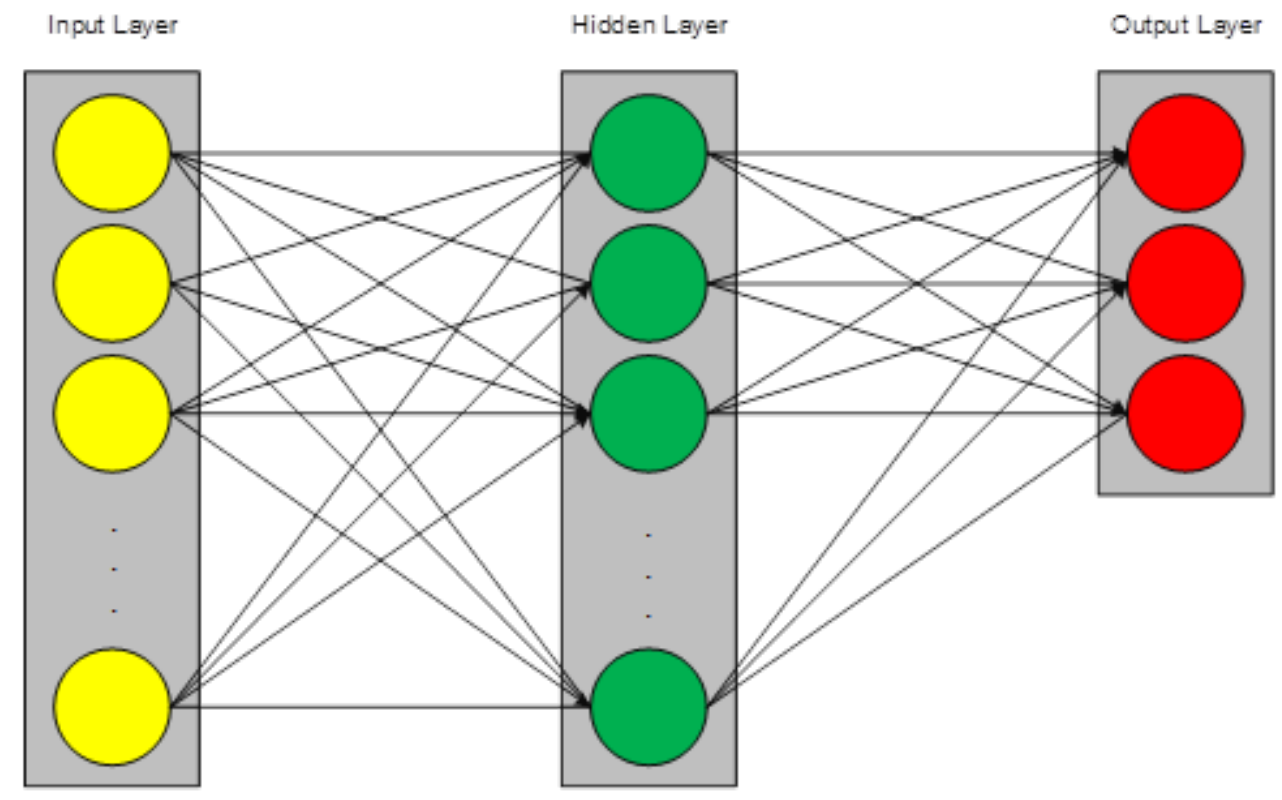

Figure 4.2: A sample structure of an artificial neural network

\subsubsection{Batch Normalization Layer}

Since deep networks can be sensitive to the weights initialization and other configurations, training deep networks with multiple layers is challenging. The distribution of the inputs to layers in the network may change due to various issues. 
Batch normalization is a technique for standardizing the inputs to each neural network layer. It also accelerates the training process and reduces the generalization error. This process can be done using the mean and standard deviation of the input variable to a layer in each mini-batch. Equation 4-3 is used for normalizing the input data to each layer.

$$
\bar{x}_{\imath}=\frac{x_{i}-\mu_{B}}{\sqrt{\sigma_{B}^{2}+\varepsilon}}
$$

Where $\bar{x}_{l}$ is the normalized vector, $x_{i}$ is the original vector, $\mu_{B}$ is the batch mean, and $\sigma_{B}^{2}$ is the batch variance.

\subsubsection{Activation Layer}

Activation functions are added into neural networks in order to add nonlinearity into the model. This will help the model to learn more complex relationships in the data. The most common activation functions are listed below.

\subsubsection{Sigmoid}

This activation function is computationally expensive, not zero-centered, and also causes vanishing gradient problems. Therefore, it is not usually used in real models. The sigmoid is defined as,

$$
\sigma(x)=\frac{1}{1+e^{-x}}
$$

\subsubsection{Tanh}

Although the problem of not being zero-centered is solved in this activation function, it still kills the gradient when the neuron is saturated. It is defined as,

$$
f(x)=\tanh (x)
$$

\subsubsection{Rectified Linear Unit (ReLU)}

This function does not saturate in the positive region. It is computationally efficient and converges faster than sigmoid/ tanh in practice. Also, ReLU overcomes the vanishing gradient problem, allowing models to learn faster and perform better. However, it is not zero-centered, and also the gradient for negative values is always zero. It is defined as,

$$
f(x)=\max (0, x)
$$




\subsubsection{Leaky $\operatorname{ReLU}$}

It has all the advantages of previous functions and covers all their drawbacks. It is defined as,

$$
f(x)=\max (0.1 x, x)
$$

\subsubsection{Maxout}

It generalizes ReLU and Leaky ReLU. Also, it does not die nor saturate. Nevertheless, it doubles the number of parameters. Therefore, it is expected to take more time for the model to learn.

$$
f(x)=\max \left(w_{1}^{T} x+b_{1}, w_{2}^{T} x+b_{2}\right)
$$

\subsubsection{Exponential Linear Units (ELU)}

It has all the benefits of ReLU. Also, it does not die and is closer to zero mean outputs. However, it is more expensive computationally. It is defined as,

$$
f(x)=\left\{\begin{array}{cl}
x, & x \geq 0 \\
\alpha\left(e^{x}-1\right), & x<0
\end{array}\right.
$$

Based on the advantages of ReLU and its robustness, it is selected as the activation function for this study.

\subsubsection{Optimizer}

Optimizers help neural networks in minimizing the loss by changing the parameters such as weights and learning rate. Neurons weights are initialized using some strategies and are updated with each epoch using the following equation.

$$
W_{\text {new }}=W_{\text {old }}-\text { learning rate } \times\left(\nabla_{w} L\right)_{W_{\text {old }}}
$$

\subsubsection{Gradient Descent (GD)}

It is one of the most basic optimization algorithms. In this algorithm, weights are updated using Eq. 4-10. Although it is easy to implement, it takes an entire dataset to compute the derivative to update the old weights. This process requires a lot of memory and computational resources. Also, it is vulnerable to getting stuck at local minima or saddle points. 


\subsubsection{Stochastic Gradient Descent (SGD)}

In order to overcome the expensive computations of GD, SGD is suggested as an extension of GD. Instead of considering the whole dataset at a time, the SGD algorithm derivative is computed, taking one point at a time. It needs less computational resources than GD; however, it takes more time to converge. The problem of getting stuck at local minima still exists in this algorithm.

\subsubsection{Mini-Batch Stochastic Gradient Descent (MB-SGD)}

This algorithm overcomes the problem of the significant time of converging in the SGD algorithm. It uses a small batch of the dataset to compute derivate. After many iterations, it is observed that the MB-SGD and GD have almost the same value for derivate the loss function. Although it takes less time to converge than SGD, the updates are much noisier compared to the GD algorithm.

\subsubsection{SGD with momentum}

It covers the disadvantages of noisy updates in the MB-SGD algorithm by denoising the gradients. It uses an exponential weighting average, giving more weightage to recent updates than the previous updates. It has all the advantages of the SGD algorithm and also converges faster than GD. However, it needs to compute one more variable in each epoch.

\subsubsection{Adaptive Gradient (AdaGrad)}

In all the previous algorithms, the learning rate remains constant; however, in the AdaGrad algorithm, the learning rate for weights updating will be decreasing with the number of iterations. This algorithm does not need to update the learning rate manually. However, as the number of iterations grows, the learning rate decreases, causing slow convergence.

\subsubsection{Adaptive Moment Estimation (Adam)}

It stores an exponentially decaying average of past gradients in addition to keeping an exponentially decaying average of past squared gradients like the AdaDelta algorithm. Among the discussed optimization algorithms, it has the best performance, although it is computationally expensive. Adam works with momentums of first and second order. In addition to storing an exponentially decaying average of past squared gradients, it keeps an exponentially decaying average of past gradients. Using averages makes the algorithm converge towards the minima at a faster pace.

Adam is relatively easy to configure, and the default configuration parameters do well in most situations. Since it combines the best properties of the AdaGrad and RMSProp algorithms, it can handle sparse gradients on noisy problems. Based on the significant 
advantages of Adam over other optimizers, it is selected as the optimizer algorithm for this study.

\subsection{HYPERPARAMETERS}

The network hyperparameters, such as number of layers and number of neurons in each layer, were selected based on preliminary experiments. The final model configuration is comprised of an input layer, multiple hidden layers with various neurons in each, and an output layer. Figure 4.3 shows the structure of the proposed model. The model was evaluated as described in the following.

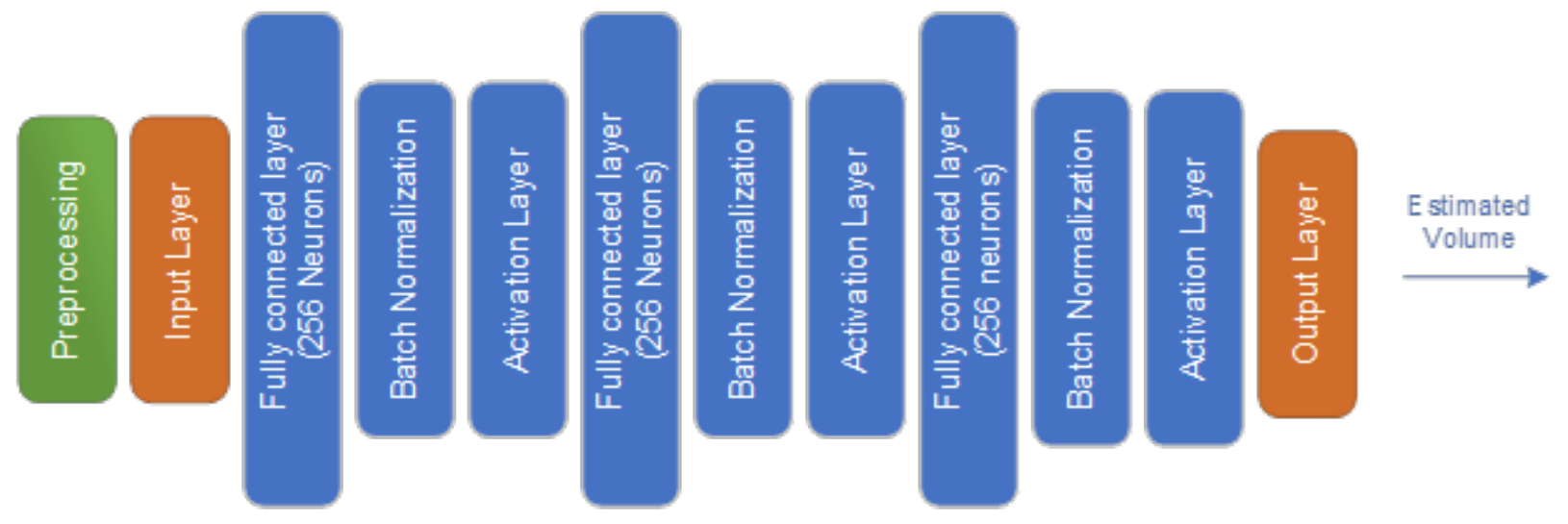

Figure 4.3: Structure of the Fully Connected Neural Network

The hyperparameters are tuned to maximize model performance on the validation dataset. The parameters used in model training are shown in Table 4.1.

Table 4.1: Considered Training Parameters Based on Hyperparameter Tuning

Hyperparameters

Number of Hidden Layers

Number of Neurons In Each Layer

Batch Size

Maximum Epoch

Learning Rate

Optimizer

\section{Value}

3

$256 / 256 / 256$

500

20

$1 \mathrm{e}-3$

Adam

The pseudocode of the proposed is given in Figure 4.4. 


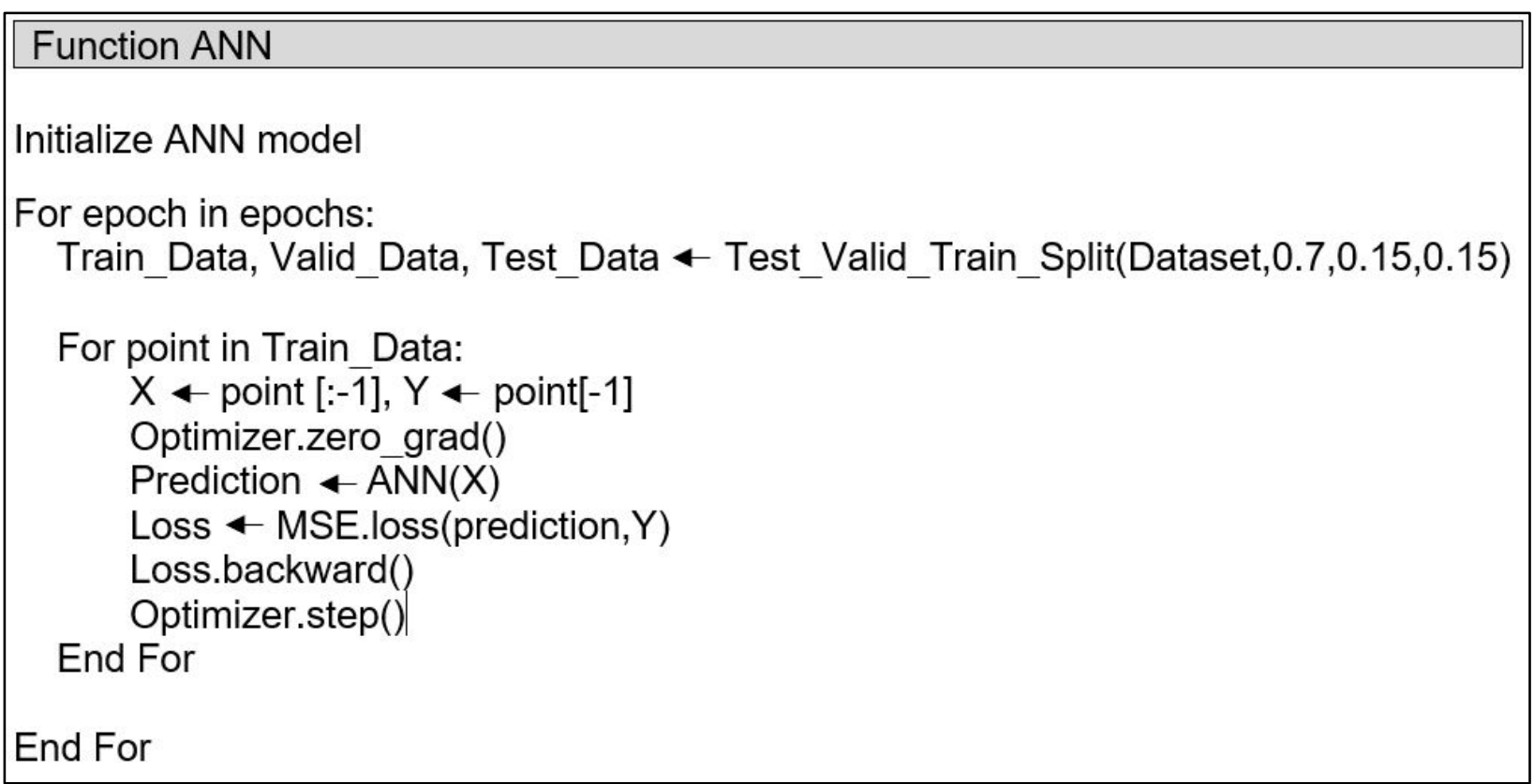

Figure 4.4: ANN Pseudocode

\subsection{EVALUATION METHODS}

The output of the proposed model is the traffic volume at each time and specific location. The performance of the model could be evaluated using the metrics discussed below.

\subsubsection{Coefficient of Determination $\left(R^{2}\right)$}

This measure shows the proportion of traffic volume variance that the model explains. The $R^{2}$ metric is computed as,

$$
R^{2}=1-\frac{\sum_{j=1}^{n}\left(y_{j}-\hat{y}_{j}\right)^{2}}{\sum_{j=1}^{n}\left(y_{j}-\bar{y}_{j}\right)^{2}}
$$

where $y_{j}$ denotes the actual traffic volume, $\hat{y}_{j}$ is the model prediction, and $\bar{y}$ represents the sample average. Values closer to 1 indicate better model performance.

\subsubsection{Mean Absolute Percentage Error (MAPE)}

This measure represents the relative accuracy of the model. It is defined as, 


$$
M A P E=\left(\frac{1}{n} \sum_{j=1}^{n}\left|\frac{\hat{y}_{j}-y_{j}}{y_{j}}\right|\right) \times 100
$$

It varies between 0 and $100 \%$. Smaller values indicate better model performance.

\subsubsection{Mean Squared Error (MSE)}

The MSE metric measures the average of the squared errors. It is defined as,

$$
M S E=\sum_{i=1}^{n} \frac{\left(\hat{y}_{j}-y_{j}\right)^{2}}{n}
$$

The smaller the MSE, the better the performance is.

\subsubsection{Root Mean Square Error (RMSE)}

The RMSE metric measures the differences between the predicted value and the observed ones. It is defined as,

$$
R M S E=\sqrt{\sum_{i=1}^{n} \frac{\left(\hat{y}_{j}-y_{j}\right)^{2}}{n}}
$$

A model with a smaller RMSE has a better performance compared to the other models.

\subsubsection{Mean Absolute Error (MAE)}

It measures the difference between two continuous variables. It is defined as,

$$
M A E=\sum_{i=1}^{n} \frac{\left|y_{j}-\hat{y}_{j}\right|}{n}
$$

The smaller MAE means that the predicted values are closer to the observed value than the larger MAE.

\subsection{Research Flow chart}

The whole process of traffic estimation is shown in Figure 4.5. 


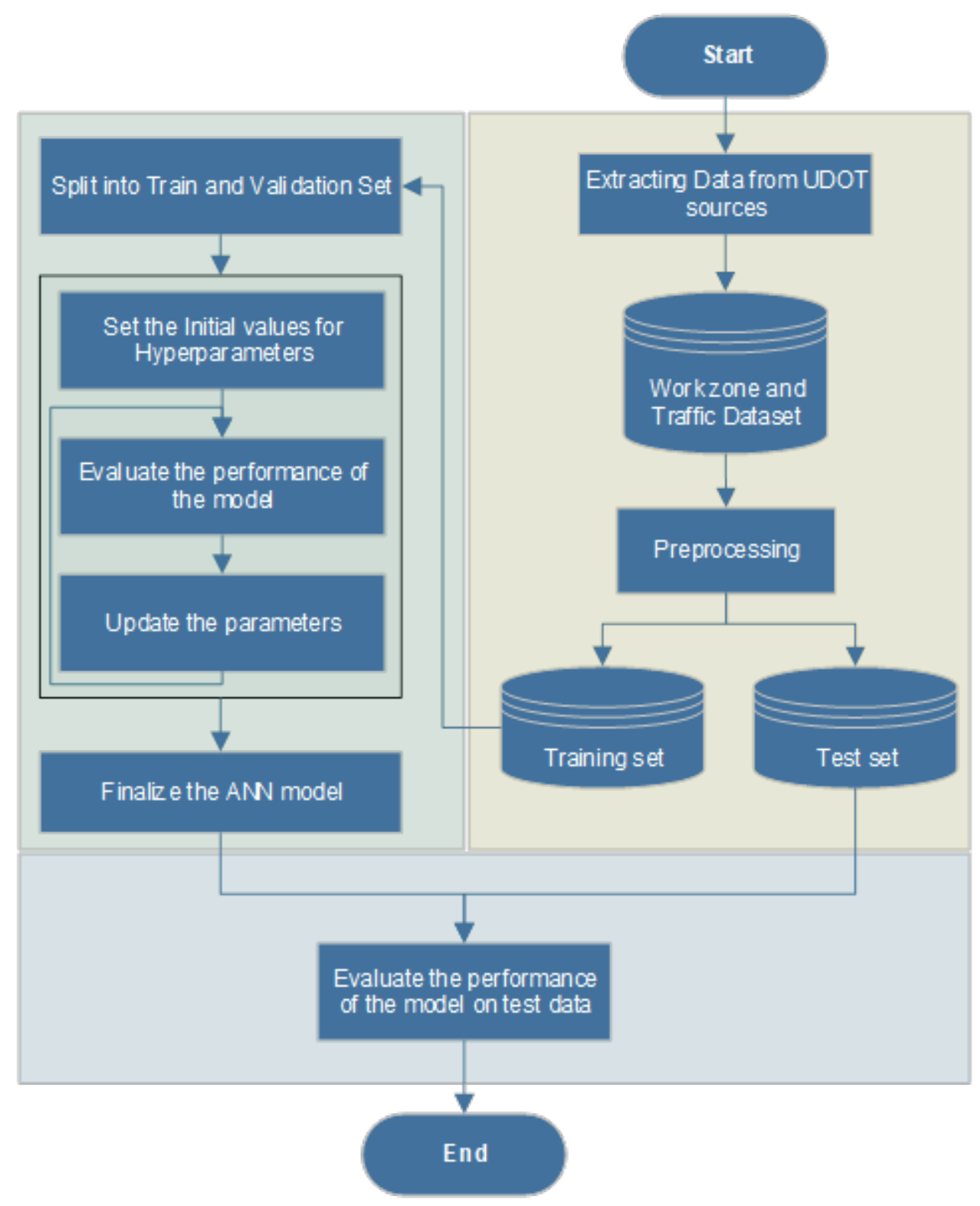

Figure 4.5: Work Zone Traffic Estimation Methodology 


\subsection{RESULTS}

\subsection{SUMMARY}

In this study, the traffic dataset is randomly divided into three groups: $70 \%$ as training data, $15 \%$ as validation data, and $15 \%$ as test data, respectively. The hyperparameters have been tuned using the validation set and the model performance. Then, the best model has been applied to the test set. Performance of the proposed ANN model is determined using four different measures. Figure 5.1 shows the loss diagram and R2 score for training, validation, and test set for a batch size of 200.
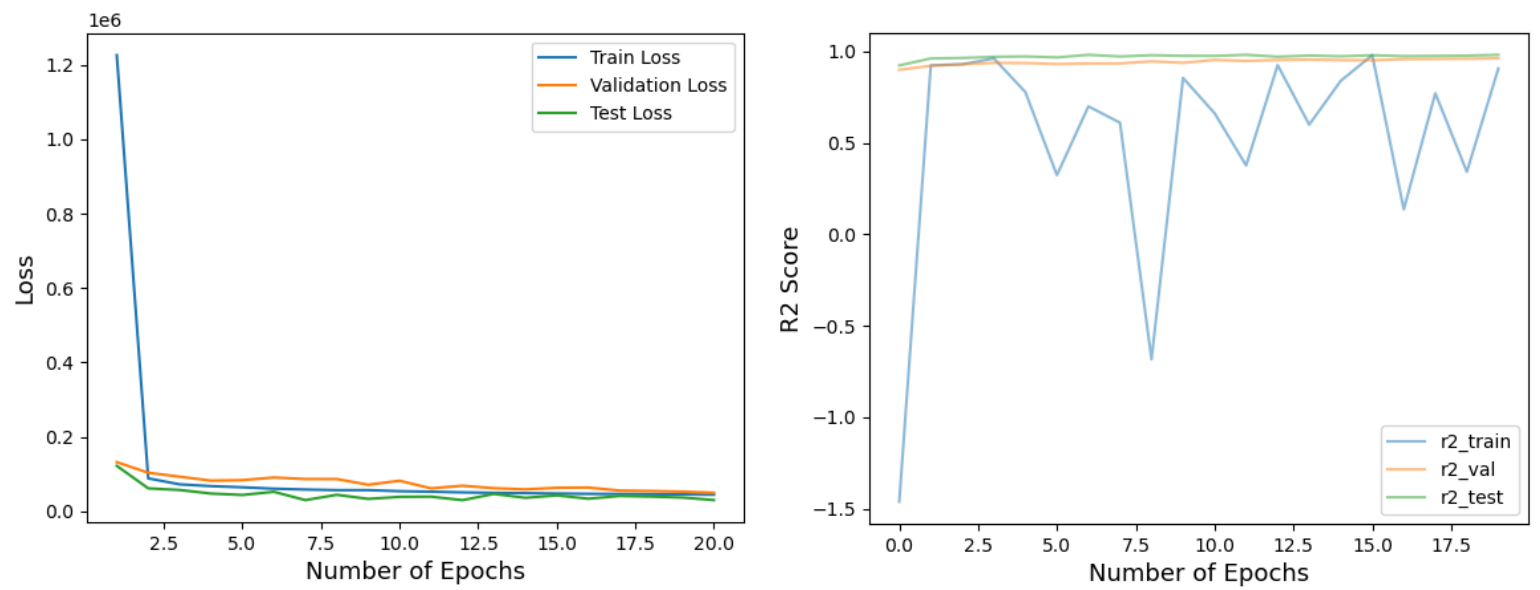

Figure 5.1: Loss Function and R2 score diagram for 20 Epochs and Batch Size of 200

As shown in Figure 5.1, all three loss diagrams decrease with increases in epochs, meaning that the choice of learning rate is acceptable. Also, the R2 score increases as the number of epochs increases. Other evaluation metrics diagrams are shown in Figure 5.2.
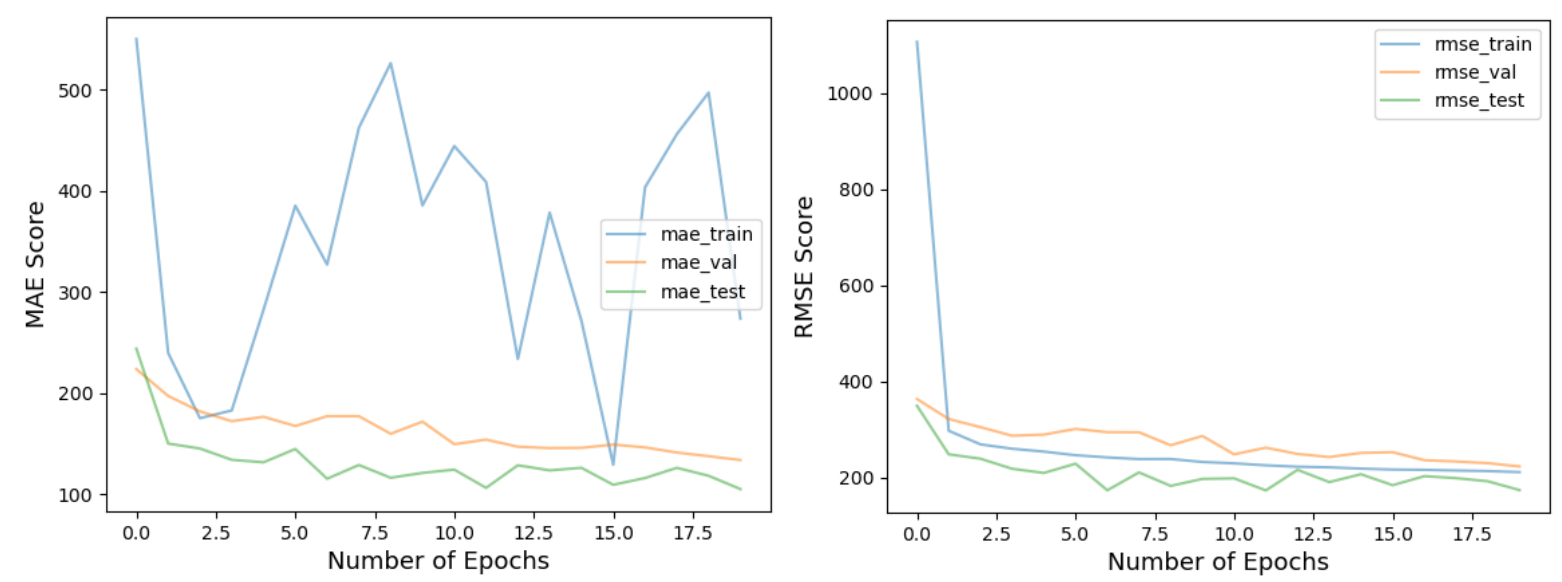

Figure 5.2: MAE and RMSE metrics for 20 Epochs and Batch Size of 200 
As shown in Figure 5.2, there are many fluctuations in the R2 score and MAE score. In order to investigate the effect of batch size, the batch size is increased to 500 .

Comparing the loss value of the $20^{\text {th }}$ epoch shows that it outperforms the previous model with a batch size of 200. Figure 5.3 shows the loss diagram and R2 score of training, validation, and test set for a batch size of 500 and 20 epochs.
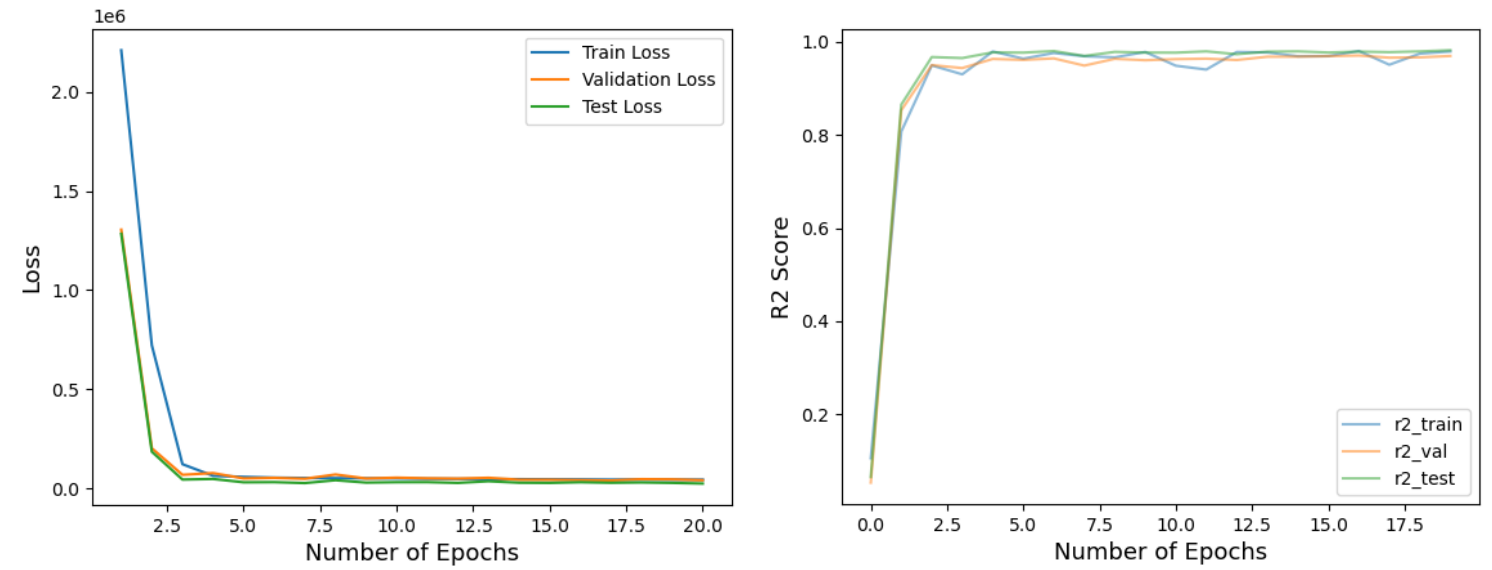

Figure 5.3: Loss Function and R2 score diagram for 20 Epochs and Batch Size of 500

Other evaluation metrics are shown in Figure 5.4.
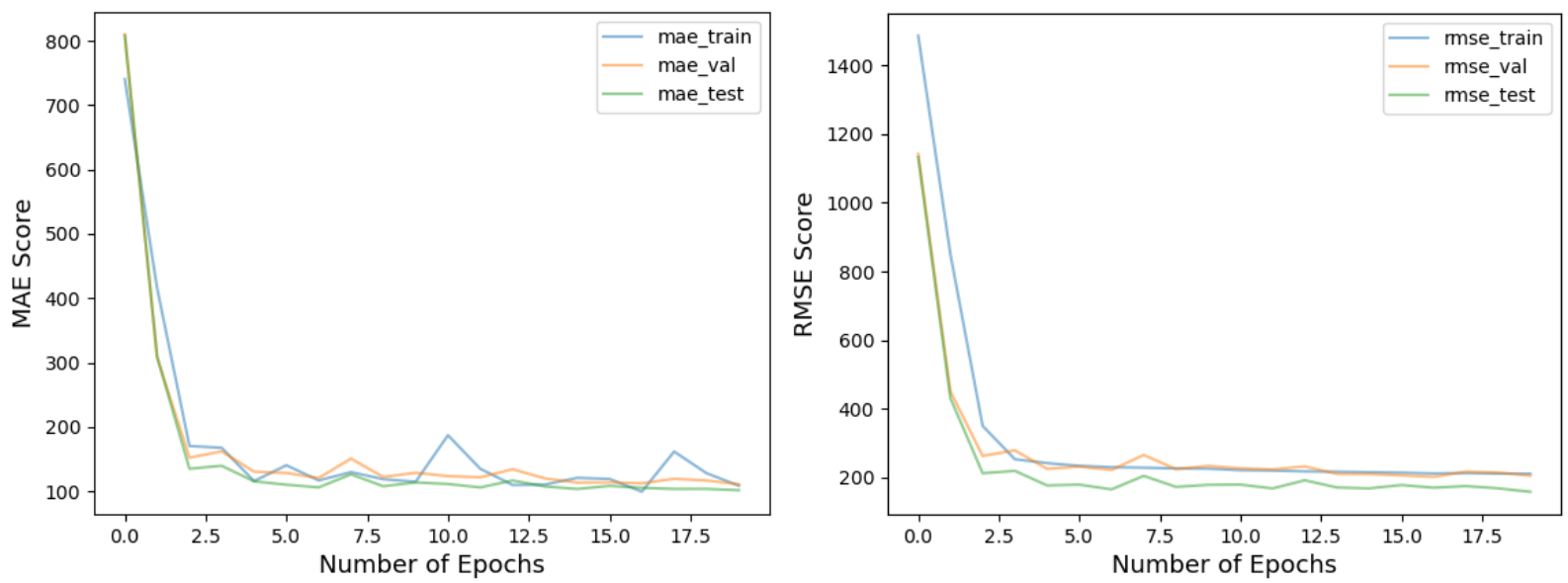

Figure 5.4: MAE and RMSE metrics for 20 Epochs and Batch Size of 500

A smaller value of train loss than the test loss shows that the model is trained better in comparison to the previous model. Since the volume of the validation set is much smaller than the training and test set, the estimated values are more reliable with less value of losses. The loss value of all three divided datasets is decreasing with an increase in the number of epochs. Therefore, the model is trained perfectly without any possibility of overfitting. As shown in Figure 5.3, there are fewer fluctuations in the R2 and MAE diagram showing that the model is stable and the training process was performed well. 
The data collected in this study included the field data, data acquired from open-source datasets, and the data acquired from the permanent sensors implemented at several locations on the interstate highways. The model evaluation for a random seed showed the model outperformance with an R2, RMSE, and MAE being $0.98,158$, and 101, respectively. Determining the study results more precisely shows that the model has much higher accuracy in those locations with more considerable traffic volume. However, the model could not yield acceptable results in zones with fewer traffic volumes. One possible justification for that could be the higher number of data points with higher traffic values, which could cause the network to be optimized based on those values. The MAE shows that the average absolute difference between the observed values and the predicted values is 101 vehicles per hour, meaning that the actual traffic is in the range of \pm (Predicted Values +101$)$.

Since the data is randomly sampled for train, validation, and test sets, it is difficult to visualize the model performance for a specific station at a given time. However, to make a graphical comparison of how the model predictions match the ground truth data, Figure 5.5 shows 50 randomly selected data points.

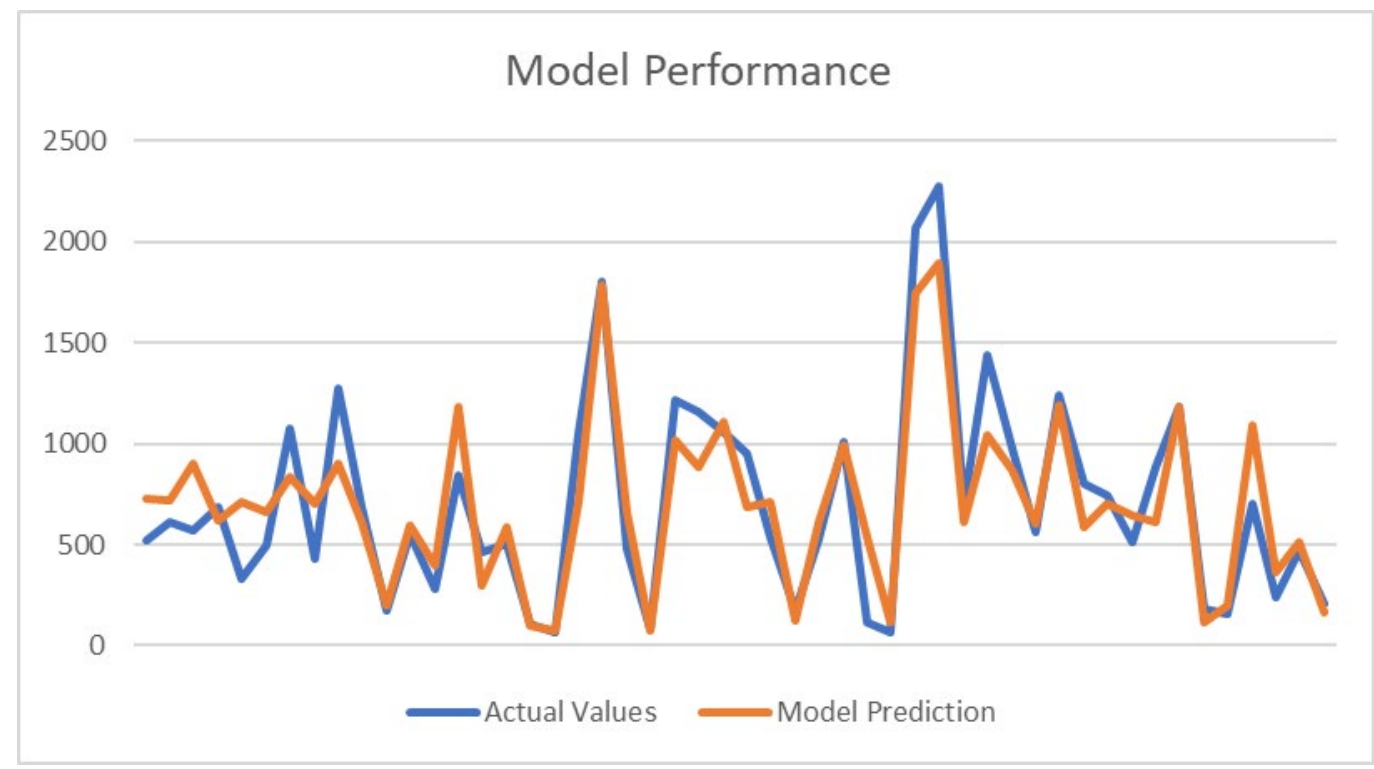

Figure 5.5: Graphical comparison of the model performance and the actual data 


\subsection{CONCLUSION \\ 6.1 SUMMARY}

Construction work zones are one of the main reasons for traffic delays and road congestion. This happens due to a decrease in available lanes, imposed speed limits, and narrower lanes. The availability of reliable and accurate traffic flow prediction models can save time and cost. In this study, an artificial neural network model is developed to predict hourly traffic volume in a statewide network based on the data collected by Utah transportation agencies. The developed model is based on the project data, road data, and traffic features such as lane width, heavy vehicle percentage, work intensity, road slope, work-zone length, project duration, year, season, day of the week, and time of day. The main benefit of the proposed model is that it does not require users to set various adjustment factors based on practical experience. The presented model would bring insights for having more accurate traffic prediction in work zones.

The suggested neural network model is trained and evaluated on around 400,000 data points collected from about 80 projects on Utah roadways. The developed model is trained on $70 \%$ of the data and is evaluated using the other $30 \%$, divided into $15 \%$ of validation and $15 \%$ of test set. The main goal of this project is to estimate historical hourly volumes throughout the state of Utah. The results show that there exists a strong correlation between the predicted and actual values. Specifically, numerical results of four random seeds show consistent outperformance of the proposed model, with an average R2, RMSE, and MAE being $0.98,101$, and 158 , respectively.

Due to the limited number of traffic sensors on state roads, it is almost impossible to estimate the work zones' traffic flow on the roads without traffic sensors. The developed deep neural network examined the feasibility of using historical data to develop a datadriven tool for evaluating traffic impacts of work zones based on work zone and spatiotemporal features. The accuracy of all types of work zone results, including short and long term, day and night time, and interstate and arterial work zones, were acceptable, with under $2 \%$ error in the predicted traffic volume. The developed model makes predictions of traffic volume within work zones using 20 input variables. One important point is that the project focused on state work zones, not meaning it is applicable to other cities and countries.

\subsection{LIMITATIONS AND RECOMMENDATIONS}

One of the main limitations of this study is the lack of exact information on the project location, project time, and lane closure strategies. For example, one project may have happened during nighttime when there is much less traffic. However, those that occurred during the daytime have more traffic impacts. Additionally, not all of the project lengths have been occupied by work zones. Many road projects are happening in 
multiple segments due to limited available heavy equipment. This could further affect the traffic conditions on the roads.

The model performance could be assessed using other machine learning models, such as random forest, support vector machine (SVM), and XGBoost. XGBoost is an optimized boosting algorithm that uses the gradient boosting framework. Boosting algorithms solve data science problems using a parallel decision-tree structure. Due to the importance of work zone traffic prediction, many studies have applied various machine learning models to investigate the effect of work zones on road traffic. The results of the proposed model could be compared to the work zone capacity estimation models existing in the literature.

Based on the encouraging results of this project, further studies could be carried out using the probe vehicle data to improve the model's reliability by decreasing the RMSE, MAPE, and MAE values. Also, the model could be used in navigation systems to improve the route choice of drivers. Thanks to recent advancements in computing technologies, computer vision can facilitate the process of automatically detecting work zone features and extracting them using different techniques such as photogrammetry [56], [57]. A valuable resource for work zone detection and feature extraction on roadsides are the videos and images collected by Mandli. Mandli is a specialized highway data collection company that integrates 3D pavement technology, mobile LiDAR, and geospatial equipment for multiple DOTs throughout the U.S. 


\section{REFERENCES}

[1] D. Schrank, B. Eisele, and T. Lomax, "2019 Urban Mobility Report," 2019.

[2] FHWA, "FHWA Operations - Reducing Recurring Congestion." https://ops.fhwa.dot.gov/program_areas/reduce-non-cong.htm (accessed May 20, 2021).

[3] R. Li, Y. Hu, and Q. Liang, "T2F-LSTM Method for Long-Term Traffic Volume Prediction," IEEE Transactions on Fuzzy Systems, vol. 28, no. 12, pp. 3256-3264, 2020.

[4] D. Zhang and M. R. Kabuka, "Combining weather condition data to predict traffic flow: a GRU-based deep learning approach," IET Intelligent Transport Systems, vol. 12, no. 7, pp. 578-585, 2018.

[5] A. H. Mashhadi, M. Farhadmanesh, A. Rashidi, and N. Marković, "Review of Methods for Estimating Construction Work Zone Capacity," Transportation Research Record, p. 03611981211002202, 2021.

[6] A. H. Mashhadi, M. Farhadmanesh, A. Rashidi, and N. Marković, "State-of-the-Art Methods in Estimating Freeway Work zones Capacity: A Literature Review," Transportation Research Board 100th Annual MeetingTransportation Research Board, no. TRBAM-21-01863, 2021.

[7] R. A. Krammes and G. O. Lopez, Updated capacity values for short-term freeway work zone lane closures, no. 1442. 1994.

[8] S. Racha, M. Chowdhury, W. Sarasua, and Y. Ma, "Analysis of work zone traffic behavior for planning applications," Transportation Planning and Technology, vol. 31, no. 2, pp. 183-199, 2008.

[9] A. Al-Kaisy and F. Hall, "Guidelines for estimating capacity at freeway reconstruction zones," Journal of Transportation Engineering, vol. 129, no. 5, pp. 572-577, 2003.

[10] J. Weng and X. Yan, "New methodology to determine work zone capacity distribution," Transportation Research Record, vol. 2461, no. 1, pp. 25-31, 2014.

[11] J. Weng and X. Yan, "Probability distribution-based model for work zone capacity prediction," Journal of Advanced Transportation, vol. 50, no. 2, pp. 165-179, 2016.

[12] J. Weng, Y. Yu, and L. Ma, "Uncertainty-based prediction of work zone capacity using a Bayesian approach," in Proceedings of the Institution of Civil EngineersTransport, 2019, vol. 172, no. 1, pp. 24-35.

[13] W. v Ping and K. Zhu, "Evaluation of work zone capacity estimation models: A computer simulation study," 2006.

[14] I. Chatterjee, P. Edara, S. Menneni, and C. Sun, "Replication of work zone capacity values in a simulation model," Transportation research record, vol. 2130, no. 1, pp. 138-148, 2009.

[15] X. Wen, "A work zone simulation model for travel time prediction in a connected vehicle environment," arXiv preprint arXiv:1801.07579, 2018.

[16] A. K. Das and U. Chattaraj, "Work zone traffic simulation using cellular automata," European transport: international journal of transport economics, engineering \& law, vol. 74, pp. e4-e4, 2019. 
[17] C. Yeom, N. M. Rouphail, W. Rasdorf, and B. J. Schroeder, "Simulation guidance for calibration of freeway lane closure capacity," Transportation Research Record, vol. 2553, no. 1, pp. 82-89, 2016.

[18] X. D. Kan, H. Ramezani, and R. F. Benekohal, "Calibration of VISSIM for freeway work zones with time-varying capacity," 2014.

[19] B. Park and H. Qi, "Microscopic simulation model calibration and validation for freeway work zone network-a case study of VISSIM," in 2006 IEEE Intelligent Transportation Systems Conference, 2006, pp. 1471-1476.

[20] J. Weng and Q. Meng, "Decision Tree-Based Model for Estimation of Work Zone Capacity," Transportation research record, vol. 2257, no. 1, pp. 40-50, 2011.

[21] J. Weng and Q. Meng, "Ensemble tree approach to estimating work zone capacity," Transportation research record, vol. 2286, no. 1, pp. 56-67, 2012.

[22] H. Adeli and X. Jiang, "Neuro-fuzzy logic model for freeway work zone capacity estimation," Journal of Transportation Engineering, vol. 129, no. 5, pp. 484-493, 2003.

[23] A. Karim and H. Adeli, "Radial basis function neural network for work zone capacity and queue estimation," Journal of Transportation Engineering, vol. 129, no. 5, pp. 494-503, 2003.

[24] Y. Hou, P. Edara, and C. Sun, "Traffic flow forecasting for urban work zones," IEEE transactions on intelligent transportation systems, vol. 16, no. 4, pp. 1761-1770, 2014.

[25] Z. Bian and K. Ozbay, "Estimating Uncertainty of Work Zone Capacity using Neural Network Models," Transportation Research Record, vol. 2673, no. 2, pp. 49-59, 2019, doi: 10.1177/0361198118825136.

[26] Y. Chen, M. Guizani, Y. Zhang, L. Wang, N. Crespi, and G. M. Lee, "When traffic flow prediction meets wireless big data analytics," arXiv preprint arXiv:1709.08024, 2017.

[27] J. Weng and Q. Meng, "Estimating capacity and traffic delay in work zones: An overview," Transportation Research Part C: Emerging Technologies, vol. 35, pp. 34-45, 2013.

[28] J. J. Wang and C. M. Abrams, "Planning and Scheduling Work Zone Traffic Control," 1981.

[29] C. L. Dudek and S. H. Richards, "Traffic capacity through work zones on urban freeways," Texas Transportation Institute, Texas A \& M University System, 1981.

[30] K. K. Dixon, "Capacity and delay for North Carolina freeway work zones." North Carolina State University, 1995.

[31] K. K. Dixon, J. E. Hummer, and A. R. Lorscheider, "Capacity for North Carolina freeway work zones," Transportation Research Record, vol. 1529, no. 1, pp. 2734, 1996.

[32] J. Yi, "Traffic characteristics and estimation of traffic delays and user costs at Indiana freeway work zones," Indiana. Dept. of Transportation, 1999.

[33] A. Al-Kaisy, M. Zhou, and F. Hall, "New insights into freeway capacity at work zones: Empirical case study," Transportation Research Record, vol. 1710, no. 1, pp. 154-160, 2000.

[34] T. Maze, S. D. Schrock, and A. Kamyab, "Capacity of freeway work zone lane closures," work, vol. 6, no. 8, p. 12, 2000. 
[35] T. Kim, D. J. Lovell, and J. Paracha, "A new methodology to estimate capacity for freeway work zones," 2001.

[36] S. Venugopal and A. Tarko, "Investigation of factors affecting capacity at rural freeway work zones," 2001.

[37] R. F. Benekohal, A.-Z. Kaja-Mohideen, and M. v Chitturi, "Evaluation of construction work zone operational issues: Capacity, queue, and delay," Publication ITRC FR 00/01-4. Illinois Transportation Research Center, 2003.

[38] R. F. Benekohal, A.-Z. Kaja-Mohideen, and M. v Chitturi, "Methodology for estimating operating speed and capacity in work zones," Transportation Research Record, vol. 1883, no. 1, pp. 103-111, 2004.

[39] W. A. Sarasua, W. J. Davis, D. B. Clarke, J. Kottapally, and P. Mulukutla, "Evaluation of interstate highway capacity for short-term work zone lane closures," Transportation Research Record, vol. 1877, no. 1, pp. 85-94, 2004.

[40] D. F. Arguea, "Simulation-based approach to estimate the capacity of a temporary freeway work zone lane closure." University of Florida Gainesville, FL, USA, 2006.

[41] W. A. Sarasua, W. J. Davis, M. A. Chowdhury, and J. H. Ogle, "Estimating interstate highway capacity for short-term work zone lane closures: development of methodology," Transportation research record, vol. 1948, no. 1, pp. 45-57, 2006.

[42] C. Hicks, I. Shaikh, B. McCabe, and S. L. Tighe, "Measuring Work Zone Capacity, Part I," 2009.

[43] C. Hicks, I. Shaikh, B. McCabe, and S. Tighe, "Measuring Work Zone CapacityPart 2," 2009.

[44] R. G. Batson et al., "Work zone lane closure analysis model.," University Transportation Center for Alabama, 2009.

[45] T. Notbohm, A. Drakopoulos, and A. Dehman, "Freeway work zone lane capacity.," 2009.

[46] K. Heaslip, A. Kondyli, D. Arguea, L. Elefteriadou, and F. Sullivan, "Estimation of freeway work zone capacity through simulation and field data," Transportation research record, vol. 2130, no. 1, pp. 16-24, 2009.

[47] G. H. Bham and S. H. Khazraee, "Missouri Work Zone Capacity: Results of Field Data Analysis [TPF-5-(081)]," Missouri University of Science and Technology. Dept. of Civil, Architectural ..., 2011.

[48] L. A. Ortiz, "Highway work zone capacity estimation using field data from Kansas." Kansas State University, 2014.

[49] J. Weng and Q. Meng, "Incorporating work zone configuration factors into speedflow and capacity models," Journal of advanced transportation, vol. 49, no. 3, pp. 371-384, 2015.

[50] N. von der Heiden and J. Geistefeldt, "Capacity of freeway work zones in Germany," Transportation research procedia, vol. 15, pp. 233-244, 2016.

[51] P. Strömgren and J. Olstam, "A model for capacity reduction at roadwork zone," Transportation research procedia, vol. 15, pp. 245-256, 2016.

[52] J. Bae, K. Choi, and J. H. Oh, "Multicontextual machine-learning approach to modeling traffic impact of urban highway work zones," Transportation Research Record, vol. 2645, no. 1, pp. 184-194, 2017. 
[53] C. Lu, J. Dong, A. Sharma, T. Huang, and S. Knickerbocker, "Predicting freeway work zone capacity distribution based on logistic speed-density models," Journal of Advanced Transportation, vol. 2018, 2018.

[54] Z. Bian and K. Ozbay, "Estimating uncertainty of work zone capacity using neural network models," Transportation research record, vol. 2673, no. 2, pp. 49-59, 2019.

[55] "What is CUDA? Parallel programming for GPUs | InfoWorld." https://www.infoworld.com/article/3299703/what-is-cuda-parallel-programming-forgpus.html (accessed Jul. 21, 2021).

[56] M. Farhadmanesh, C. Cross, A. H. Mashhadi, A. Rashidi, and J. Wempen, "Highway Asset and Pavement Condition Management using Mobile Photogrammetry," Transportation Research Record, p. 03611981211001855, 2021.

[57] M. Farhadmanesh, C. Cross, A. H. Mashhadi, A. Rashidi, and J. Wempen, "Use of Mobile Photogrammetry Method for Highway Asset Management," Transportation Research Board 100th Annual MeetingTransportation Research Board, no. TRBAM-21-01864, 2021. 